\title{
Spectra associated to symmetric monoidal bicategories
}

\author{
ANGÉLICA M OSORNO
}

\begin{abstract}
We show how to construct a $\Gamma$-bicategory from a symmetric monoidal bicategory and use that to show that the classifying space is an infinite loop space upon group completion. We also show a way to relate this construction to the classic $\Gamma$-category construction for a permutative category. As an example, we use this machinery to construct a delooping of the $\mathcal{K}$-theory of a rig category as defined by Baas, Dundas and Rognes [2].
\end{abstract}

18D05, 55B20, 55P42; 19D23, 55N15

\section{Introduction}

Symmetric monoidal bicategories appear in many contexts in mathematics. One example is Bimod, the bicategory of rings, bimodules and homomorphisms of bimodules, with tensor product as the monoidal structure. Another example is $n C o b$, the bicategory of closed $n$-manifolds, cobordisms and diffeomorphisms between cobordisms, with disjoint union as the monoidal structure. The definition of symmetric monoidal bicategory is cumbersome. Some incomplete definitions can be found in McCrudden [19] and Day and Street [5], while the most concise and complete definition can be found in Schommer-Pries [24]. Shulman [27] shows how to obtain symmetric monoidal bicategories from symmetric monoidal double categories, which are easier to understand.

In view of the importance of the construction of spectra from symmetric monoidal categories (see May [17] and Segal [25]), we can ask if there is a similar construction for symmetric monoidal bicategories. More specifically, we would like to know if the group completion of the classifying space of a symmetric monoidal bicategory is an infinite loop space. In this paper we show that this is the case for strict symmetric monoidal bicategories. To do this, we use Segal's theory of $\Gamma$-spaces [25]. Our procedure gives a functor from symmetric monoidal bicategories to spectra that we call $\mathbb{A}$. This functor is analogous to the functor $\mathbb{E}$ from symmetric monoidal categories to spectra, as developed by Elmendorff and Mandell [6].

Baas, Dundas and Rognes [2] defined the $\mathcal{K}$-theory of a rig category. A rig category is a category that roughly behaves like a ring with no additive inverses. Given a rig 
category $\mathcal{R}$, we construct the bicategory of $\mathcal{R}$-modules, $\mathcal{G} \mathcal{L}(\mathcal{R})$. It turns out that the $\mathcal{K}$-theory space of $\mathcal{R}$ is the group completion of the classifying space of $\mathcal{G} \mathcal{L}(\mathcal{R})$. We prove that $\mathcal{G L}(\mathcal{R})$ is a strict symmetric monoidal bicategory, and hence, $\mathcal{K}(\mathcal{R})$ is an infinite loop space. We compare this result with those of Baas, Dundas, Richter and Rognes [1], where the authors obtain an equivalence of spaces

$$
\mathcal{K}(\mathcal{R}) \simeq K(\mathbb{E} \mathcal{R})
$$

where the right-hand side is the infinite loop space associated to the algebraic $K$-theory spectrum of the ring spectrum $\mathbb{E} \mathcal{R}$. We prove that this equivalence is an equivalence of infinite loop spaces. See Theorem 4.7 for the precise statement.

The paper is organized as follows. Section 1 contains the necessary background information on bicategories and $\Gamma$-spaces. Section 2 includes the statements of the main results. Section 3 is dedicated to the application of the main results to the $\mathcal{K}-$ theory of rig categories, in particular, we show that the $\mathcal{K}$-theory of a rig category is an infinite loop space. In Section 4, we recall the construction of the ring spectrum $\mathbb{E} \mathcal{R}$ and show that there is a map of infinite loop spaces between $\mathcal{K}(\mathcal{R})$ and $K(\mathbb{E} \mathcal{R})$. This section also contains some material on symmetric spectra in the category of categories that is needed for some of the constructions. Section 5 contains the main proofs and constructions. Appendix A gives an account of the construction of the classifying space of a bicategory.

The results from this paper are part of the author's PhD thesis under the supervision of Mark Behrens. We would like to thank Peter May for suggesting the current organization and for his many comments on earlier versions of this paper. Finally, we would like to thank the referee, for his very detailed report and many suggestions for improvement.

\section{Background}

\subsection{Bicategories}

In this paper we will be working with bicategories. For definitions and proofs of the basic theorems, we refer the reader to the early papers of Bénabou [3] and Street [28] on bicategories and some more recent accounts by Leinster [13] and Street [29].

Throughout the document we will assume categories and bicategories are enriched over Top, the category of compactly generated Hausdorff spaces, without explicitly saying it. A bicategory is enriched over topological spaces if all the categories of morphisms are enriched. We do this so that we can accommodate the main example of $V_{e c t}$, which has a natural topology on the sets of morphisms $G L_{n}(\mathbb{C})$. 
Given a bicategory $\mathcal{C}$, we will denote the vertical composition by $\circ$, and the horizontal composition by $*$. For an object $A$ in $\mathcal{C}, I_{A}$ denotes the identity 1-morphism. For a 1 -morphism $f, \operatorname{id}_{f}$ denotes the identity 2 -morphism. The associativity isomorphism is denoted by $a$, while the right and left identity isomorphisms are denoted by $r$ and $l$, respectively. We use the term 2-category to refer to the strict version, that is, a bicategory where the natural isomorphisms $a, r$ and $l$ are all identities.

Let $F: \mathcal{C} \rightarrow \mathcal{D}$ be a pseudofunctor. We denote the functoriality 2 -isomorphisms by

$$
F_{f, g}^{2}: F(g) * F(f) \rightarrow F(g * f) \quad \text { and } \quad F_{A}^{0}: I_{F A} \rightarrow F\left(I_{A}\right) .
$$

These are subject to coherence axioms as stated in [29, page 566]. A pseudofunctor is said to be strict if the 2-isomorphisms $F_{f, g}^{2}$ and $F_{A}^{0}$ are the identity.

\section{$1.2 \Gamma$-spaces}

Segal's $\Gamma$-spaces give an infinite loop space machine. In [25], it is shown that a symmetric monoidal category $\mathcal{M}$ gives rise to a $\Gamma$-space, and hence to a connective spectrum, whose zero space is the group completion of the classifying space of $\mathcal{M}$. The connection between symmetric monoidal categories and connective spectra was made precise by Thomason [31], proving that there is an equivalence of categories between a suitably defined stable homotopy category of symmetric monoidal categories and the stable homotopy category of connective spectra. This result has been recently reproved by Mandell [15].

We will recall briefly what a $\Gamma$-space is, since the definition will play a central role in the paper.

Let $\mathcal{F}$ denote the skeletal category of finite pointed sets and pointed maps. This category has as objects the sets $\mathbf{n}_{+}=\{0,1, \ldots, n\}$, for $n \geq 0$. Here 0 is the basepoint.

For $1 \leq k \leq n$, we define $i_{k}: \mathbf{n}_{+} \rightarrow \mathbf{1}_{+}$as

$$
i_{k}(j)= \begin{cases}0 & \text { if } j \neq k \\ 1 & \text { if } j=k\end{cases}
$$

Let $T o p_{*}$ be the category of compactly generated Hausdorff spaces with a basepoint.

Definition 1.1 A $\Gamma$-space $X$ is a functor $X: \mathcal{F} \rightarrow T o p_{*}$. We say $X$ is special if the map

$$
p_{n}: X\left(\mathbf{n}_{+}\right) \rightarrow X\left(\mathbf{1}_{+}\right)^{\times n}
$$

obtained by assembling the maps $i_{k}$ is a weak equivalence for all $n \geq 0$. 
The conditions in the definition above roughly imply that the space $X\left(\mathbf{1}_{+}\right)$has a multiplication that is associative and commutative up to coherent higher homotopies. The precise statement in given by the following theorem:

Theorem 1.2 [25, Proposition 1.4] Let $X$ be a special $\Gamma$-space. Then $X\left(\mathbf{1}_{+}\right)$is an infinite loop space upon group completion.

Segal and May [17] show how to construct a $\Gamma$-category from a symmetric monoidal category enriched over topological spaces, thus getting an infinite delooping of the classifying space of a symmetric monoidal category. As we mention in the introduction, given a permutative category $\mathcal{M}$, we denote the associated spectrum by $\mathbb{E}(\mathcal{M})$. We will follow a similar approach in the context of bicategories.

\subsection{Symmetric monoidal bicategories}

In broad terms, a symmetric monoidal bicategory is a bicategory with a product pseudofunctor that is associative, unital, and commutative up to coherent natural equivalences. The precise definition is quite involved, as one would imagine. For a precise definition and a historical account of the theory of symmetric monoidal bicategories we refer the reader to Schommer-Pries [24]. Incomplete versions of the definition can be found in McCrudden [19] (definition of sylleptic monoidal bicategory) and Day and Street [5] (definition of symmetric Gray monoid). Shulman [27] provides a way of constructing examples of symmetric monoidal bicategories.

In this paper we will be working with a strict version of symmetric monoidal bicategories, and that is what we will define here.

Recall that a transformation $\eta$ between pseudofunctors $F, G: \mathcal{C} \rightarrow \mathcal{D}$ consists of a 1 -morphism $\eta_{A}: \mathcal{F} A \rightarrow \mathcal{G} A$ for each $A \in \mathrm{Ob}(\mathcal{C})$, and for every pair $A, B$, natural 2-isomorphisms

$$
\eta_{f}^{2}: \mathcal{G} f * \eta_{A} \Rightarrow \eta_{B} * \mathcal{F} f
$$

The latter must satisfy some coherence conditions (see [29, page 568]).

Definition 1.3 A strict symmetric monoidal bicategory $(\mathcal{M}, \boxplus, 0, \beta)$ consists of the following data:

- a bicategory $\mathcal{M}$;

- a strict functor of bicategories $\boxplus: \mathcal{M} \times \mathcal{M} \rightarrow \mathcal{M}$;

- an object 0 in $\mathcal{M}$ called the unit;

- a transformation $\beta$ : $\boxplus \rightarrow \boxplus \circ \tau$, where $\tau$ denotes the twist pseudofunctor $\mathcal{M} \times \mathcal{M} \rightarrow \mathcal{M} \times \mathcal{M}$. 
The monoidal product $\boxplus$ is required to be strictly associative, and 0 is a strict unit, the following diagrams must commute:
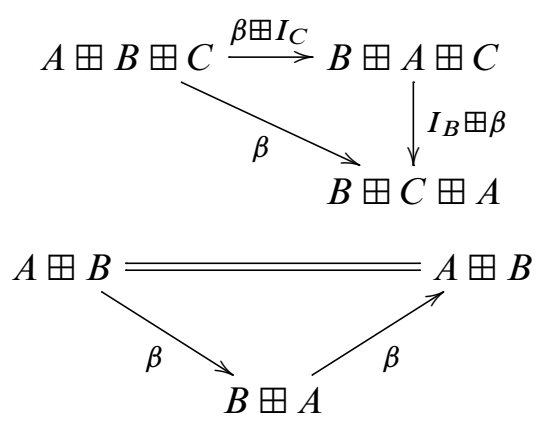

Remark 1.4 The strict version defined above is just one of the many ways in which one can strictify the notion of symmetric monoidal bicategory. In fact, there are different levels of strictness one could consider. We choose this level because it is convenient to work with and it covers the applications we have in mind. It is easy to check that this definition is a special case of the general definition of symmetric monoidal bicategory. It is not yet known whether any symmetric monoidal bicategory can be strictified to a strict symmetric monoidal bicategory as defined here.

It is known that any monoidal bicategory is equivalent to a Gray monoid by Gordon, Power and Street [7], which is a 2-category with a certain product pseudofunctor, which is strictly associative and unital. The extra data needed for the monoidal bicategory to be symmetric is carried across the equivalence but does not necessarily get any stricter.

The symmetric monoidal structure on a bicategory translates into an $H$-space structure on its classifying space (see Appendix A for details on the construction of the classifying space of a bicategory). In the case of symmetric monoidal categories, we know that we obtain an infinite loop space structure upon group completion. To show that this is also the case for (strict) symmetric monoidal bicategories we will be using Segal's $\Gamma$-space machine in the context of bicategories.

Let Bicat $_{*}$ denote the category of (small) pointed bicategories and pseudofunctors that preserve the base object.

Definition 1.5 A pseudofunctor $\mathcal{F}: \mathcal{C} \rightarrow \mathcal{D}$ is an equivalence of bicategories if there exists a pseudofunctor $\mathcal{G}: \mathcal{D} \rightarrow \mathcal{C}$ and natural equivalences, ie weakly invertible transformations,

$$
\operatorname{id}_{\mathcal{C}} \simeq \mathcal{G} \circ \mathcal{F} \quad \text { and } \quad \operatorname{id}_{\mathcal{D}} \simeq \mathcal{G} \circ \mathcal{F}
$$

Other authors use the term biequivalence to refer to the definition above. 
Definition 1.6 A $\Gamma$-bicategory $\mathcal{A}$ is a functor $\mathcal{A}: \mathcal{F} \rightarrow$ Bicat $_{*}$. We say $\mathcal{A}$ is special if the map

$$
p_{n}: \mathcal{A}\left(\mathbf{n}_{+}\right) \rightarrow \mathcal{A}\left(\mathbf{1}_{+}\right)^{\times n}
$$

is an equivalence of bicategories for all $n \geq 0$.

This definition is analogous to that of a special $\Gamma$-space, with the connection made clear by the following lemma, where $|\mathbf{N}(-)|$ denotes the classifying space functor as constructed in Appendix A.

Lemma 1.7 Let $\mathcal{A}$ be a special $\Gamma$-bicategory. Then $|\mathbf{N} \mathcal{A}|: \mathcal{F} \rightarrow$ Top $_{*}$ is a special $\Gamma$-space.

Proof The classifying space functor $|\mathbf{N}(-)|:$ Bicat $\rightarrow$ Top preserves products and sends equivalences of bicategories to homotopy equivalences of spaces (see Proposition A.4).

\section{Statement of results}

We are now ready to state the main results of the paper. The proofs of both these theorems are delayed until Section 5.

Theorem 2.1 Let $\mathcal{M}$ be a (strict) symmetric monoidal bicategory. Then there is a special $\Gamma$-bicategory $\mathbb{W} \mathcal{M}$ such that

$$
(\mathbb{W} \mathcal{M})\left(\mathbf{1}_{+}\right) \cong \mathcal{M}
$$

Therefore the classifying space $|\mathbf{N} \mathcal{M}|$ is an infinite loop space upon group completion.

Using this theorem, we can construct a functor

$$
\text { A: SymMonBicat } \longrightarrow \text { Spectra. }
$$

This functor will be used in Section 3 to construct the $\mathcal{K}$-theory of rig categories.

We will compare the $\Gamma$-bicategory construction to the standard $\Gamma$-category construction; see May [17] and Shimada and Shimakawa [26]. The construction studied in these papers gives a functor

$$
\mathbb{U}: \text { Perm } \longrightarrow \Gamma \text { Cat },
$$

from the category of permutative categories to the category of $\Gamma$-categories. 
Given a 2-category $\mathcal{M}$, let $T \mathcal{M}$ denote the category enriched over topological spaces obtained from $\mathcal{M}$ as follows: the objects are the same as the objects of $\mathcal{M}$ and the space of morphisms $T \mathcal{M}(X, Y)$ is the classifying space of the category $\mathcal{M}(X, Y)$. If $\mathcal{M}$ is a strict symmetric monoidal 2 -category such that the braiding is a strict natural isomorphism, then $T \mathcal{M}$ is a permutative topological category.

Theorem 2.2 Let $\mathcal{M}$ be a strict symmetric monoidal 2-category such that the braiding is a strict natural isomorphism. Then there is a special $\Gamma-(2$-category) $\mathbb{V} \mathcal{M}$ and a levelwise equivalence

$$
\mathbb{V} \mathcal{M} \longrightarrow \mathbb{W} \mathcal{M}
$$

of special $\Gamma$-bicategories. Furthermore, there is a levelwise isomorphism

$$
\mathbb{U} T \mathcal{M} \longrightarrow T \mathbb{V} \mathcal{M}
$$

of $\Gamma$-categories.

By [4, Theorem 6.4], the geometric realization of the 2-nerve of a strict 2-category $\mathcal{C}$ is equivalent to the classifying space of the category $T \mathcal{C}$ (see Appendix A for details). Applying this to $\Gamma-(2-$ categories $)$ levelwise, we get the middle equivalence in the following corollary.

Corollary 2.3 Let $\mathcal{M}$ be a strict symmetric monoidal 2-category. Then there are levelwise equivalences of $\Gamma$-spaces

$$
B \mathbb{U} T \mathcal{M} \simeq B T \mathbb{V} \mathcal{M} \simeq|\mathbf{N} \mathbb{V} \mathcal{M}| \simeq|\mathbf{N} \mathbb{W} \mathcal{M}|
$$

\section{Delooping $K$-theory of rig categories}

Baas, Dundas and Rognes [2] introduce the notions of $2 K$-theory and 2-vector bundles as a way to categorify topological $K$-theory and vector bundles. One of their objectives is to define a cohomology theory of a geometric nature that has chromatic level 2.

In general they define the $K$-theory space of a (commutative) rig category $\mathcal{R}$ in terms of a bar construction for monoidal categories and show there is an equivalence of spaces

$$
\mathcal{K}(\mathcal{R}) \simeq K(\mathbb{E} \mathcal{R})
$$

between the $K$-theory of the rig category $\mathcal{R}$ and the algebraic $K$-theory space of the ring spectrum $\mathbb{E} \mathcal{R}$. 


\subsection{Definition of $K$-theory of a rig category}

The notion of a rig category (also known as a bimonoidal category) is obtained by categorifying the notion of a ring without negatives. Here is a categorically incomplete definition.

Definition 3.2 A rig category $\left(\mathcal{R}, \oplus, 0, \gamma_{\oplus}, \otimes, 1, \delta\right)$ consists of a permutative category $\left(\mathcal{R}, \oplus, 0, \gamma_{\oplus}\right)$, together with a strict monoidal structure $(\otimes, 1)$, such that right distributivity and nullity of zero hold strictly, and there is a left distributivity natural isomorphism

$$
\delta: a \otimes(b \oplus c) \rightarrow(a \otimes b) \oplus(a \otimes c) .
$$

A bipermutative category $\mathcal{R}$ is a rig category that is permutative under $(\otimes, 1)$, with commutativity isomorphism $\gamma_{\otimes}$.

In the commutative case, the coherence diagrams necessary for a precise definition of the less strict notion of a symmetric bimonoidal category were given by Laplaza [12], and the coherence diagrams for a nonsymmetric bimonoidal category can be found, for example, in Guillou [8, Definition 3.1]. By May [16, Proposition VI.3.5], any symmetric bimonoidal category is equivalent to a bipermutative category. Similarly, by [8, Theorem 1.2], any bimonoidal category is equivalent to a rig category.

Let $\left(\mathcal{R}, \oplus, 0, c_{\oplus}, \otimes, 1, \delta\right)$ be a rig category. Then, as in [2], we can define $M_{n}(\mathcal{R})$, the category of $n \times n$ matrices over $\mathcal{R}$. Its objects are matrices $V=\left(V_{i, j}\right)_{i, j=1}^{n}$ whose entries are objects of $\mathcal{R}$. The morphisms are matrices $\phi=\left(\phi_{i, j}\right)_{i, j=1}^{n}$ of isomorphisms in $\mathcal{R}$, such that the source (resp, target) of $\phi_{i, j}$ is the $(i, j)$-entry of the source (target) of $\phi$. As a category, $M_{n}(\mathcal{R})$ is isomorphic to $\mathcal{R}^{n \times n}$.

Moreover, $M_{n}(\mathcal{R})$ is a monoidal category, with multiplication

$$
M_{n}(\mathcal{R}) \times M_{n}(\mathcal{R}) \stackrel{\dot{\rightarrow}}{\rightarrow} M_{n}(\mathcal{R})
$$

given by sending the pair $(U, V)$ to

$$
W_{i k}=\bigoplus_{j=1}^{n} U_{i j} \otimes V_{j k} .
$$

Since $\oplus$ is strictly associative, there is no ambiguity.

This multiplication has a unit object $I_{n}$, given by the matrix with 1 in the diagonal and 0 elsewhere. The objects 0 and 1 are strict units for $\oplus$ and $\otimes$ respectively, and the nullity of 0 holds strictly, so $I_{n}$ is a strict unit as well. 
Proposition 3.3 [2,3.3] Matrix multiplication makes $\left(M_{n}(\mathcal{R}), \cdot, I_{n}\right)$ into a monoidal category.

The natural associativity isomorphism

$$
a: U \cdot(V \cdot W) \rightarrow(U \cdot V) \cdot W
$$

is given by entry-wise use of $\gamma_{\oplus}$ and $\delta$.

Recall that if $R$ is a semiring, $G L_{n}(R)$ is the subgroup of $M_{n}(R)$ that contains all the matrices which are invertible as matrices over the ring completion $G r_{+}(R)$. The following definition is also taken from [2].

Definition 3.4 Let $G L_{n}(\mathcal{R}) \subset M_{n}(\mathcal{R})$ be the full subcategory of matrices $V=$ $\left(V_{i, j}\right)_{i, j=1}^{n}$ whose matrix of path components lies in $G L_{n}\left(\pi_{0}(\mathcal{R})\right)$. We call $G L_{n}(\mathcal{R})$ the category of weakly invertible matrices. By convention we will let $G L_{0}(\mathcal{R})=\underline{1}$ be the unit category, with one object and one morphism.

Note that $G L_{n}(\mathcal{R})$ inherits a monoidal structure from $M_{n}(\mathcal{R})$.

Given a monoidal category $\mathcal{C}$, the authors in [2] define a bar construction for monoidal categories, B.C , which is a simplicial object in $C a t$. As we point out in Remark A.2, this definition coincides with the 2 -nerve of the bicategory $\Sigma \mathcal{C}$, that is, the bicategory with one object whose category of morphisms is given by $\mathcal{C}$.

We note that block sum of matrices in $\mathcal{R}$ makes

$$
\coprod_{n \geq 0}\left|\mathbf{B} \bullet G L_{n}(\mathcal{R})\right|
$$

into a topological monoid, since it is strictly associative and unital. Hence we define the $K$-theory of $\mathcal{R}$ to be the group completion

$$
\mathcal{K}(\mathcal{R}):=\Omega B\left(\coprod_{n \geq 0}\left|\mathbf{B}_{\bullet} G L_{n}(\mathcal{R})\right|\right) .
$$

The motivation behind the definition of $K$-theory for rig categories comes from the categorification of complex $K$-theory. As we know well, the complex $K$-theory space classifies virtual vector bundles.

A 2-vector space, as defined by Kapranov and Voevodsky [9], is a category equivalent to $\left(\text { Vect }_{\mathbb{C}}\right)^{n}$ for some $n$. Heuristically, this should be thought of as a module category over Vect $\mathbb{C}$. In [2], the authors introduce the notion of a complex 2-vector bundle over a topological space and construct a classifying space for these bundles. A 2-vector 
bundle is roughly a bundle of 2 -vector spaces over $X$, defined in terms of transition functions, which are given by matrices of vector spaces. For the precise definition we refer the reader to [2, Section 2].

One of the main results in [2] is that the stable equivalence classes of virtual 2-vector bundles over a space $X$ are in one-to-one correspondence with homotopy classes of maps from $X$ to $\mathcal{K}\left(\right.$ Vect $\left._{\mathbb{C}}\right)$, where Vect $_{\mathbb{C}}$ is a considered as a rig category using direct sum and tensor product.

\section{2 $K$-Theory as a classifying space of a bicategory}

We now define the bicategory $\mathcal{G} \mathcal{L}(\mathcal{R})$, whose classifying space can be used to construct $\mathcal{K}(\mathcal{R})$.

Definition 3.5 Let $\mathcal{G} \mathcal{L}(\mathcal{R})$ be the bicategory of finite dimensional free modules over $\mathcal{R}$, defined as follows. The objects are labeled by the natural numbers $\underline{n} \geq 0$. Given objects $\underline{n}, \underline{m}$, the category of morphisms is

$$
\mathcal{G L}(\mathcal{R})(\underline{n}, \underline{m})= \begin{cases}G L_{n}(\mathcal{R}) & \text { if } n=m, \\ \varnothing & \text { if } n \neq m .\end{cases}
$$

and the composition is given by matrix multiplication. In other words,

$$
\mathcal{G L}(\mathcal{R})=\coprod_{n \geq 0} \Sigma G L_{n}(\mathcal{R})
$$

With this identification, the identity 1 -morphism for the object $\underline{n}$ is given by the identity matrix $I_{n}$.

Example 3.6 Let Vect $_{k}$ be the commutative rig category of vector spaces over the field $k$. Then $\mathcal{G} \mathcal{L}\left(\right.$ Vect $\left._{k}\right)$ is a sub-bicategory of the bicategory of 2 -vector spaces defined by Kapranov and Voevodsky [9]. The 1-morphisms are matrices of vector spaces such that their matrices of dimensions have determinant \pm 1 .

We can use the bicategory $\mathcal{G} \mathcal{L}(\mathcal{R})$ to give an alternative definition of the $K$-theory of $\mathcal{R}$. We have the following identifications of simplicial categories:

$$
\coprod_{n \geq 0} \mathbf{B} . G L_{n}(\mathcal{R})=\coprod_{n \geq 0} \mathbf{N} \Sigma G L_{n}(\mathcal{R})=\mathbf{N}\left(\coprod_{n \geq 0} \Sigma G L_{n}(\mathcal{R})\right)=\mathbf{N} \mathcal{G} \mathcal{L}(\mathcal{R}),
$$

where $\mathbf{N}(-)$ denotes the $2-$ nerve (see Appendix A). Hence, we can redefine the $K$-theory space

$$
\mathcal{K}(\mathcal{R}):=\Omega B|\mathbf{N G \mathcal { L }}(\mathcal{R})|
$$


This is the definition we will use in the following sections. In the next section we will show that the multiplication on $|\mathbf{N} \mathcal{G} \mathcal{L}(\mathcal{R})|$ comes from a strict functor

$$
\mathcal{G L}(\mathcal{R}) \times \mathcal{G} \mathcal{L}(\mathcal{R}) \rightarrow \mathcal{G L}(\mathcal{R})
$$

which will give $\mathcal{G} \mathcal{L}(\mathcal{R})$ the structure of a strict symmetric monoidal bicategory.

\subsection{Symmetric monoidal structure on the bicategory $\mathcal{G} \mathcal{L}(\mathcal{R})$}

Just as we can take direct sum of modules over a ring, we can take direct sum of modules over a rig category. This will provide $\mathcal{G L}(\mathcal{R})$ with a symmetric monoidal structure, which in turn will give rise to an infinite delooping of $\mathcal{K}(\mathcal{R})$.

Theorem 3.7 The bicategory $\mathcal{G L}(\mathcal{R})$ is strict symmetric monoidal with the monoidal operation given by block sum of matrices:

$$
\begin{aligned}
\boxplus: \mathcal{G L}(\mathcal{R}) \times \mathcal{G} \mathcal{L}(\mathcal{R}) & \rightarrow \mathcal{G L}(\mathcal{R}) \\
(\underline{n}, \underline{m}) & \mapsto \underline{n+m} \\
(U, V) & \mapsto\left[\begin{array}{c|c}
U & 0 \\
\hline 0 & V
\end{array}\right] \\
(\varphi, \psi) & \mapsto\left[\begin{array}{l|l}
\varphi & 0 \\
\hline 0 & \psi
\end{array}\right] .
\end{aligned}
$$

The matrix [0] is the matrix with all entries equal to 0 , the unit of $\oplus$ in $\mathcal{R}$.

Proof We first note that the operation described above gives a strict functor of bicategories, since it preserves the identity and the composition:

$$
\begin{aligned}
& I_{n} \boxplus I_{m}=I_{n+m}, \\
& {\left[\begin{array}{c|c}
U^{\prime} & 0 \\
\hline 0 & V^{\prime}
\end{array}\right] *\left[\begin{array}{c|c}
U & 0 \\
\hline 0 & V
\end{array}\right]=\left[\begin{array}{c|c}
U^{\prime} * U & 0 \\
\hline 0 & V^{\prime} * V
\end{array}\right] . }
\end{aligned}
$$

The second equation holds because of the strict nullity and unity of 0 in $\mathcal{R}$. The unit of $\boxplus$ is $\underline{0}$. We note that for $U \in G L_{n}(\mathcal{R}), V \in G L_{m}(\mathcal{R})$ and $W \in G L_{p}(\mathcal{R})$,

$$
\begin{gathered}
(U \boxplus V) \boxplus W=U \boxplus(V \boxplus W), \\
I_{0} \boxplus U=U=U \boxplus I_{0} .
\end{gathered}
$$

The natural equivalence $\beta_{n, m}: \underline{n} \boxplus \underline{m} \rightarrow \underline{m} \boxplus \underline{n}$ is given by the block matrix

$$
\left[\begin{array}{c|c}
0 & I_{m} \\
\hline I_{n} & 0
\end{array}\right] .
$$


Since 0 and 1 are strict units in $\mathcal{R}$, for $U \in G L_{n}(\mathcal{R})$ and $V \in G L_{m}(\mathcal{R})$,

$$
\beta_{n, m} *(U \boxplus V)=\left[\begin{array}{c|c}
0 & V \\
\hline U & 0
\end{array}\right]=(V \boxplus U) * \beta_{n, m},
$$

so $\beta$ is a strict transformation.

We note that $\beta_{m, n} * \beta_{n, m}=I_{n+m}$ which both implies that $\beta$ is a natural isomorphism and that it is its self-inverse. We conclude that $\mathcal{G L}(\mathcal{R})$ is a strict symmetric monoidal bicategory.

By Theorem 2.1, there exists a spectrum $\mathbb{A}(\mathcal{G L}(\mathcal{R}))$. We can thus define the $K$-theory spectrum of $\mathcal{R}$ as

$$
\mathbb{K}(\mathcal{R}):=\mathbb{A}(\mathcal{G L}(\mathcal{R})) .
$$

It is clear from the construction that the zeroth space of this spectrum is precisely the $\mathcal{K}$-theory space, as defined in Section 3.2, obtaining thus the following result.

Theorem 3.8 The $K$-theory space of the rig category $\mathcal{R}$ is an infinite loop space, with the additive structure provided by the block sum of matrices. More precisely, we can identify $\mathcal{K}(\mathcal{R})$ with the zeroth space of the spectrum $\mathbb{K}(\mathcal{R})$.

\section{Algebraic $K$-theory of $\mathbb{E} \mathcal{R}$}

In order to compare $\mathcal{K}(\mathcal{R})$ with $K(\mathbb{E} \mathcal{R})$, we must first describe the latter. Given a rig category $\mathcal{R}$, by forgetting the multiplicative structure, we can construct the spectrum $\mathbb{E} \mathcal{R}$ associated to the permutative category $(\mathcal{R}, \oplus)$. The results of $[6 ; 18]$ show that the multiplicative structure of $\mathcal{R}$ makes $\mathbb{E} \mathcal{R}$ into a ring spectrum, and furthermore, if $\mathcal{R}$ is commutative, $\mathbb{E} \mathcal{R}$ is an $E_{\infty}$ ring spectrum. Note that in $[2 ; 1]$ the authors denote $\mathbb{E} \mathcal{R}$ by $H \mathcal{R}$, pointing out the analogy to the Eilenberg-Mac Lane spectrum of a ring.

The model we are taking for the symmetric ring spectrum $\mathbb{E} \mathcal{R}$ is that of [6]. We now construct the category $\mathcal{G} \mathcal{L}(\mathbb{E} \mathcal{R})$.

Let $\mathbb{E} \mathcal{R}(p)$ denote the $p$-th space of the spectrum $\mathbb{E} \mathcal{R}$. We let

$$
M_{n}(\mathbb{E} \mathcal{R}(p))=\operatorname{Map}_{*}\left(\mathbf{n}_{+}, \mathbf{n}_{+} \wedge \mathbb{E} \mathcal{R}(p)\right) .
$$

This should be thought of as the space of $n \times n$ matrices with entries in $\mathbb{E} \mathcal{R}(p)$, such that at most one of the entries per column is not equal to the basepoint. 
Then the space of matrices

$$
M_{n}(\mathbb{E} \mathcal{R})=\underset{\mathbf{p} \in \mathcal{I}}{\operatorname{hocolim}} \Omega^{m} M_{n}(\mathbb{E} \mathcal{R}(m))
$$

is a topological monoid, as we explain shortly. The homotopy colimit here is taken over $\mathcal{I}$, which is the category of finite sets and injections. Since $M_{n}(\mathbb{E} \mathcal{R})$ is a topological monoid, then so is $G L_{n}(\mathbb{E} \mathcal{R})$, which is defined by the pullback

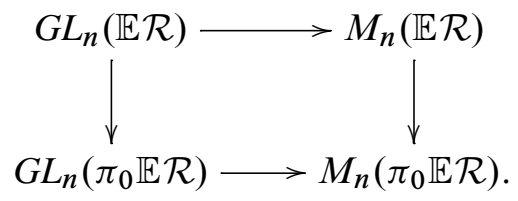

The category $\mathcal{G} \mathcal{L}(\mathbb{E} \mathcal{R})$ has as objects the natural numbers $\underline{n}$. The spaces of morphisms are given by

$$
\mathcal{G L}(\mathbb{E} \mathcal{R})(\underline{n}, \underline{m})= \begin{cases}G L_{n}(\mathbb{E} \mathcal{R}) & \text { if } n=m \\ \varnothing & \text { if } n \neq m\end{cases}
$$

Block sum of matrices makes $\mathcal{G} \mathcal{L}(\mathbb{E} \mathcal{R})$ into a permutative topological category, with associated spectrum $\mathbb{K}(\mathbb{E} \mathcal{R})$.

In order to compare the classifying spaces of $\mathcal{G L}(\mathcal{R})$ and $\mathcal{G L}(\mathbb{E} \mathcal{R})$, we use two auxiliary bicategories, and to define them, we need a theory of symmetric spectra in Cat, which we now develop.

\subsection{Symmetric spectra of categories}

We generalize Minian's definition of spectra in Cat [21, Definition 5.1] to symmetric spectra and symmetric ring spectra as follows.

We first recall some notation from [20; 21].

For pointed categories $C, D$, we denote by $C \wedge D$ the smash product, as defined in [20, Definition 2.2]. By construction, the smash product satisfies the universal property given by the isomorphism between the pointed sets of pointed functors

$$
\mathrm{Fun}_{*}(C \wedge D, E) \cong \mathrm{Fun}_{*}\left(C, \mathrm{Cat}_{*}(D, E)\right) .
$$

It is not true in general that $B(C \wedge D)$ is equivalent to $B C \wedge B D$, but there is a natural map $B C \wedge B D \rightarrow B(C \wedge D)$.

Let $\alpha \in \mathbb{N}$. The $\alpha$-circle $S_{\alpha}^{1}$ is the pointed category depicted as (in the case of $\alpha$ odd)

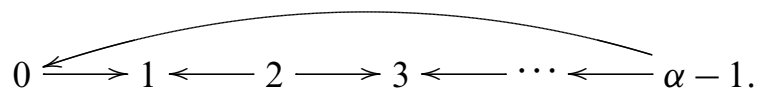

The $p$-th $\alpha$-sphere $S_{\alpha}^{p}$ is given by the iterated smash product $\left(S_{\alpha}^{1}\right)^{\wedge p}$. 
Let $C$ be a pointed category. The $\alpha$-loop category of $C$ is defined as the category of pointed functors and pointed natural transformations from $S_{\alpha}^{1}$ to $C$, that is

$$
\Omega_{\alpha} C:=\operatorname{Cat}_{*}\left(S_{\alpha}^{1}, C\right) .
$$

For $\beta \geq \alpha$ there are subdivision functors $S_{\beta}^{1} \rightarrow S_{\alpha}^{1}$ inducing functors $\Omega_{\alpha} C \rightarrow \Omega_{\beta} C$. We define the loop category of $C$ by taking the colimit over all subdivision functors:

$$
\Omega C:=\operatorname{colim}_{\alpha} \Omega_{\alpha} C \text {. }
$$

The loop category has the property that its classifying space is weakly equivalent to the loop space of the classifying space of $C$.

For $\alpha, \beta$, there is a functor $\Omega_{\alpha} C \times \Omega_{\beta} C \rightarrow \Omega_{\gamma} C$ given by concatenation of loops. Here $\gamma=\alpha+\beta$ or $\alpha+\beta+1$, depending on whether $\alpha$ is even or odd. These concatenation functors are compatible with the subdivision functors, so they give a functor

$$
\Omega C \times \Omega C \rightarrow \Omega C .
$$

Now we can define symmetric spectra in Cat.

Definition 4.1 A symmetric spectrum $C$ in $C a t$ is a sequence of pointed categories $C_{0}, C_{1}, \ldots$ together with pointed functors

$$
S_{\alpha_{p}}^{1} \wedge C_{p} \rightarrow C_{p+1}
$$

for some natural number $\alpha_{p}$, and a basepoint preserving left action of the symmetric group $\Sigma_{p}$ on $C_{p}$ such that the iterated functor

$$
\left(S_{\alpha_{q}}^{1} \wedge S_{\alpha_{q+1}}^{1} \wedge \cdots \wedge S_{\alpha_{p+q=1}}^{1}\right) \wedge C_{q} \rightarrow C_{p+q}
$$

is $\left(\Sigma_{p} \times \Sigma_{q}\right)$-equivariant for all $p \geq 1, q \geq 0$.

Definition 4.2 A symmetric ring spectrum $C$ is a symmetric spectrum together with $\left(\Sigma_{p} \times \Sigma_{q}\right)$-equivariant pairings

$$
\mu_{p, q}: C_{p} \wedge C_{q} \rightarrow C_{p+q}
$$

for all $p, q \geq 0$ satisfying the usual coherence conditions (see [23, Section 2.1]).

\subsection{The construction of symmetric spectra from permutative categories}

There are many ways of passing from special $\Gamma$-categories to symmetric spectra. Here we sketch one of them. 
Let $\mathcal{A}: \mathcal{F} \rightarrow \mathrm{Cat}_{*}$ be a special $\Gamma$-category. We now construct the symmetric spectrum $K \mathcal{A}$ in $\mathrm{Cat}$. This construction is the same as the construction in [21]. Here we point out why this is a symmetric spectrum of categories.

The $p$-th category of the spectrum is given by the realization (in Cat) of the simplicial category

$$
k \mapsto \mathcal{A}\left(\left(\mathbf{k}_{+}\right)^{\wedge p}\right) .
$$

Note that this simplicial category has a natural action of $\Sigma_{p}$ by permuting the factors in $\left(\mathbf{k}_{+}\right)^{\wedge p}$. This gives an action of $\Sigma_{p}$ on $K \mathcal{A}(p)$.

The category $K \mathcal{A}(p)$ is isomorphic to the category $B^{p} \mathcal{A}$ defined in [21]. Indeed, consider the multisimplicial category

$$
\left(k_{1}, \ldots, k_{p}\right) \mapsto \mathcal{A}\left(\mathbf{k}_{\mathbf{1}} \wedge \wedge \cdots \wedge \mathbf{k}_{\mathbf{p}_{+}}\right) .
$$

Then $K \mathcal{A}(p)$ is the realization of the diagonal simplicial category, and $B^{p} \mathcal{A}$ is the iteration of the realization one index at a time, from right to left. Using a similar strategy to [22], one can show that the two are isomorphic.

It follows by the results in [21, Section 5] that there is a functor

$$
S_{1}^{1} \wedge K \mathcal{A}(p) \rightarrow K \mathcal{A}(p+1) .
$$

The iterated functors $S_{1}^{q} \wedge K \mathcal{A}(p) \rightarrow K \mathcal{A}(p+q)$ are equivariant by construction, so $K \mathcal{A}$ is a symmetric spectrum in $\mathrm{Cat}$.

There are also may ways of passing from permutative categories to special $\Gamma$-categories. For example, as we mentioned before, we can use the construction of [17] to produce a special $\Gamma$-category $\mathbb{U C}$ from a permutative category $\mathcal{C}$. Then $K \mathbb{U C}$ is the symmetric spectrum in $C$ at associated to $\mathcal{C}$.

\subsection{The construction of symmetric ring spectra from rig categories}

In [6], the authors construct an equivalent version of the symmetric spectrum associated to a permutative category, which is better suited to handle multiplicative structure. We will use their notation for the following definition. By realization of a simplicial category, we mean the construction defined in [21, Section 3]. This construction gives a functor from simplicial categories to categories that sends level-wise equivalences to equivalences, and preserves products up to equivalence.

Let $\mathcal{C}$ be a rig category. We now define a symmetric spectrum $\hat{\mathcal{C}}$ in $C a t$. We set $\widehat{\mathcal{C}}(0)=\mathcal{C}$ and for $p>0$, we let $\widehat{\mathcal{C}}(p)$ be the realization of the simplicial category

$$
k \mapsto \overline{\mathcal{C}}(\overbrace{\mathbf{k}_{+}, \ldots, \mathbf{k}_{+}}^{p}),
$$


where $\overline{\mathcal{C}}\left(\mathbf{k}_{1+}, \ldots, \mathbf{k}_{\mathbf{p}_{+}}\right)$is the category defined in [6, Construction 4.4]. This category has an action of $\Sigma_{p}$ via permutation of the $p$ entries.

In the proof of [6, Theorem 4.6], the authors show that there is an equivalence of categories

$$
\mathbb{U C}\left(\mathbf{k}_{\mathbf{1}} \wedge \cdots \wedge \mathbf{k}_{\mathbf{p}_{+}}\right) \rightarrow \mathcal{C}^{k^{p}}
$$

and a functor

$$
\mathcal{C}^{k^{P}} \rightarrow \overline{\mathcal{C}}\left(\mathbf{k}_{+}, \ldots, \mathbf{k}_{+}\right)
$$

that is a left adjoint, giving a functor

$$
\mathbb{U C}\left(\mathbf{k}_{\mathbf{1}} \wedge \wedge \wedge \mathbf{k}_{\mathbf{p}_{+}}\right) \rightarrow \overline{\mathcal{C}}\left(\mathbf{k}_{+}, \ldots, \mathbf{k}_{+}\right)
$$

that is a strong homotopy equivalence in the sense of [21].

Thus, by [21, Proposition 4.5] there is an equivariant strong homotopy equivalence $K \cup \mathcal{C}(p) \rightarrow \widehat{\mathcal{C}}(p)$.

Since realization of a bisimplicial category can be done taking the diagonal or by realizing first in one direction and then in the other, the category $\hat{\mathcal{C}}(p+1)$ can be constructed as the realization of the simplicial category

$$
j \mapsto\left|k \mapsto \overline{\mathcal{C}}(\mathbf{j}_{+}, \overbrace{\mathbf{k}_{+}, \ldots, \mathbf{k}_{+}}^{p})\right| .
$$

Noting that $\overline{\mathcal{C}}\left(\mathbf{1}_{+}, \mathbf{i}_{\mathbf{1}_{+}}, \mathbf{i}_{\mathbf{2}+}, \ldots, \mathbf{i}_{\mathbf{p}_{+}}\right)$is isomorphic to $\overline{\mathcal{C}}\left(\mathbf{i}_{\mathbf{1}}, \mathbf{i}_{\mathbf{2}}, \ldots, \mathbf{i}_{\mathbf{p}_{+}}\right)$and using [21, Remark 3.7], we obtain a functor

$$
S_{1}^{1} \wedge \widehat{\mathcal{C}}(p) \rightarrow \widehat{\mathcal{C}}(p+1) .
$$

These functors are compatible with those for $K \mathbb{U C}$ and the iterated functors are equivariant by construction. We thus have a symmetric spectrum $\hat{\mathcal{C}}$, and a strong homotopy equivalence of symmetric spectra $K \mathbb{U C} \rightarrow \widehat{\mathcal{C}}$.

If $\mathcal{R}$ is a rig category, the multiplication induces equivariant pairings

$$
\overline{\mathcal{R}}\left(\mathbf{k}_{\mathbf{1}+}, \ldots, \mathbf{k}_{\mathbf{p}_{+}}\right) \times \overline{\mathcal{R}}\left(\mathbf{l}_{\mathbf{1}+}, \ldots, \mathbf{l}_{\mathbf{q}_{+}}\right) \rightarrow \overline{\mathcal{R}}\left(\mathbf{k}_{\mathbf{1}+}, \ldots, \mathbf{k}_{\mathbf{p}_{+}}, \mathbf{l}_{\mathbf{1}+}, \ldots, \mathbf{l}_{\mathbf{q}_{+}}\right)
$$

that are strictly associative and unital. They induce pairings on the realization

$$
\widehat{\mathcal{R}}(p) \times \hat{\mathcal{R}}(q) \rightarrow \widehat{\mathcal{R}}(p+q),
$$

also strictly associative and unital, which make $\widehat{\mathcal{R}}$ into a symmetric ring spectrum. Note that $\widehat{\mathcal{R}}(0)=\mathcal{R}$. The symmetric spectrum in Top $\mathbb{E} \mathcal{R}$ is then given by taking the classifying space levelwise. 


\subsection{I-monoids in Cat and Top}

We must explain why $M_{n}(\mathbb{E} \mathcal{R})$ and $G L_{n}(\mathcal{R})$ are topological monoids. In order to do so, we will use the theory of $\mathcal{I}$-categories and $\mathcal{I}$-monoids in $C a t$, which we explain in this section. This is analogous to the theory of $\mathcal{I}$-spaces. A good source for all the definitions and some of the main results is [23].

Let $\mathcal{I}$ be the skeletal category of finite sets and injections. Objects are given by the natural numbers, with $\mathbf{p}=\{1,2, \ldots, p\}$, and $\mathbf{0}$ the empty set. For sets $\mathbf{p}$ and $\mathbf{q}$, we take the concatenation $\mathbf{p} \sqcup \mathbf{q}$ to be the set $\mathbf{p}+\mathbf{q}$, where the first $p$ elements correspond to those of $\mathbf{p}$, and the last $q$ to those of $\mathbf{q}$. This makes $\mathcal{I}$ into a permutative category, with the braiding given by the permutation that exchanges the first $p$ elements with the last $q$.

An $\mathcal{I}$-category (analogous to $\mathcal{I}$-space) $X$ is a functor $X: \mathcal{I} \rightarrow$ Cat $_{*}$. Note that the category $\mathrm{Cat}_{*}^{\mathcal{I}}$ is symmetric monoidal, with the symmetric product $X \otimes Y$ given by the left Kan extension of $X \times Y$ along $\sqcup: \mathcal{I} \times \mathcal{I} \rightarrow \mathcal{I}$. We say $X$ is an $\mathcal{I}$-monoid if $X$ is a monoid in $\mathrm{Cat}_{*}^{\mathcal{I}}$. Unraveling this definition we get a natural transformation $\mu: X \times X \rightarrow X(-\sqcup-)$. This transformation is given by functors

$$
\mu_{p, q}: X(p) \times X(q) \rightarrow X(p+q),
$$

for all pairs $(p, q)$. The requirement that this is a monoid means that this multiplication is strictly associative and unital, in the sense that $\mu_{p, q+r} \circ\left(\mathrm{id} \times \mu_{q, r}\right)=$ $\mu_{p+q, r} \circ\left(\mu_{p, q} \times \mathrm{id}\right)$, and the inclusion of the basepoint in $X(0)$ composed with $\mu_{0, q}$ is equal to the projection $* \times X(q) \rightarrow X(q)$; and similarly for $\mu_{p, 0}$.

Now consider the Grothendieck construction $\mathcal{I} \int X$. By [30], the classifying space $B\left(\mathcal{I} \int X\right)$ is a model for the homotopy colimit of $B X$. The extra structure of the $\mathcal{I}$-monoid $X$ implies extra structure on the Grothendieck construction.

Theorem 4.3 Let $X$ be an $\mathcal{I}$-monoid in Cat. Then $\mathcal{I} \int X$ is a strict monoidal category.

Proof The objects of $\mathcal{I} \int X$ are given by pairs $(\mathbf{p}, x)$, where $\mathbf{p}$ is an object of $\mathcal{I}$ and $x$ is an object of $X(p)$. The morphisms between $(\mathbf{p}, x)$ and $\left(\mathbf{p}^{\prime}, x^{\prime}\right)$ are given by pairs $(i, f)$, where $i: \mathbf{p} \rightarrow \mathbf{p}^{\prime}$ in $\mathcal{I}$ and $f: X(i) x \rightarrow x^{\prime}$ in $X\left(p^{\prime}\right)$.

The monoidal structure is defined as

$$
\begin{aligned}
& ((\mathbf{p}, x),(\mathbf{q}, y)) \mapsto\left(\mathbf{p} \sqcup \mathbf{q}, \mu_{p, q}(x, y)\right), \\
& ((i, f),(j, g)) \mapsto\left(i \sqcup j, \mu_{p^{\prime}, q^{\prime}}(f, g)\right) .
\end{aligned}
$$

The unit of the monoidal structure is given by $(\mathbf{0}, *)$, where $*$ denotes the basepoint of $X(0)$. Since $\mu$ is associative and unital, the monoidal structure is strict. 
We also consider a weak version of $\mathcal{I}$-monoids as follows. Let $\underline{\mathrm{Cat}}_{*}^{\mathcal{I}}$ be the 2 -category of strict functors $\mathcal{I} \rightarrow \underline{\mathrm{Cat}}_{*}$, strict natural transformations and modifications, where $\underline{\mathrm{Cat}}_{*}$ is the 2-category of based categories. This is a symmetric monoidal 2-category with the same product as defined above for $\mathrm{Cat}_{*}^{\mathcal{I}}$.

A (strictly unital) $\mathcal{I}$-pseudomonoid $X$ is a (strictly unital) pseudomonoid in $\underline{\mathrm{CaI}}_{*}^{\mathcal{I}}$, as defined in [19, Section 2]. This implies that we have an $\mathcal{I}$-category $X$ together with a natural transformation $\mu$ as above and a modification $\alpha$, which on the triple $(p, q, r)$ is given by a natural transformation

$$
\alpha_{p, q, r}: \mu_{p, q+r} \circ\left(\mathrm{id} \times \mu_{q, r}\right) \rightarrow \mu_{p+q, r} \circ\left(\mu_{p, q} \times \mathrm{id}\right),
$$

satisfying the pentagon axiom. The proof of the following theorem is the same as above, with the added associativity constraint given by $\alpha$.

Theorem 4.4 Let $X$ be a strictly unital $\mathcal{I}$-pseudomonoid in Cat. Then $\mathcal{I} \int X$ is a strictly unital monoidal category.

\subsection{I-monoids coming from symmetric spectra}

Let $C$ by a symmetric spectrum in Cat. Consider the functor $\Omega^{-} C_{(-)}$from $\mathcal{I}$ into $\mathrm{Cat}_{*}$. Note that any morphism $\mathbf{p} \rightarrow \mathbf{q}$ in $\mathcal{I}$ can be factored as the standard inclusion followed by a permutation. There is a $\Sigma_{p}$ action on $\Omega_{\alpha}^{p} C_{p}=\operatorname{Cat}_{*}\left(S_{\alpha}^{p}, C_{p}\right)$ given by conjugation. We then get an action of $\Sigma_{p}$ on $\Omega^{p} C(p)$. We use this action to define the functor on permutations.

Let $i: \mathbf{p} \rightarrow \mathbf{p}+\mathbf{q}$ be the standard inclusion. We have the structure functor $S_{\beta_{p, q}}^{q} \wedge C_{p} \rightarrow$ $C_{p+q}$. Taking the adjoint, mapping into the colimit and applying $\Omega^{p}(-)$, we get a functor $\Omega^{p} C_{p} \rightarrow \Omega^{p+q} C_{p+q}$.

If $C$ is a symmetric ring spectrum, we can give $\Omega^{-} C_{(-)}$the structure of an $\mathcal{I}$-monoid. The multiplication map is given by the colimit of the maps

$$
\begin{aligned}
\Omega_{\alpha}^{p} C_{p} \times \Omega_{\alpha}^{q} C_{q} & \longrightarrow \Omega_{\alpha}^{p+q} C_{p+q} \\
\left(f: S_{\alpha}^{p} \rightarrow C_{p}, g: S_{\alpha}^{q} \rightarrow C_{q}\right) & \longmapsto\left[s \wedge t \mapsto \mu_{p, q}(f(s), g(t))\right] .
\end{aligned}
$$

\subsection{From $\mathcal{K}(\mathcal{R})$ to $K(\mathbb{E} \mathcal{R})$}

With the machinery from the previous sections, we can now describe a map $\mathcal{K}(\mathcal{R}) \rightarrow$ $K(\mathbb{E} \mathcal{R})$ which is equivalent to the one in [1], and show that it is a map of infinite loop spaces.

We construct two auxiliary bicategories, $\widetilde{\mathcal{G L}}(\mathbb{E} \mathcal{R})$ and $\overline{\mathcal{G L}}(\mathbb{E} \mathcal{R})$, as follows. 
Consider the symmetric ring spectrum in Cat, $\widehat{\mathcal{R}}$, as described in Section 4.3. Recall that $\mathbf{n}_{+}$denotes the pointed set $\{\underline{0}, 1, \ldots, n\}$. We use the same notation to denote the discrete category with $\mathbf{n}_{+}$as set of elements. Let $M_{n}(\hat{\mathcal{R}}(p))=\operatorname{Cat}_{*}\left(\mathbf{n}_{+}, \mathbf{n}_{+} \wedge \hat{\mathcal{R}}(p)\right)$, analogous to the definition for spaces. As a category, this is equivalent to $\prod_{n} \bigvee_{n} \hat{\mathcal{R}}(p)$, and as such it is a symmetric spectrum, with the structure functors inherited from those of $\hat{\mathcal{R}}$.

It is furthermore a symmetric ring spectrum, with the pairing given on

$$
(f, g) \in M_{n}(\widehat{\mathcal{R}}(p)) \times M_{n}(\widehat{\mathcal{R}}(q))
$$

by the composition

$$
\mathbf{n}_{+} \stackrel{g}{\longrightarrow} \mathbf{n}_{+} \wedge \hat{\mathcal{R}}(q) \stackrel{f \wedge \mathrm{id}}{\longrightarrow} \mathbf{n}_{+} \wedge \hat{\mathcal{R}}(p) \wedge \hat{\mathcal{R}}(q) \stackrel{\mathrm{id} \wedge \mu_{p, q}}{\longrightarrow} \mathbf{n}_{+} \wedge \hat{\mathcal{R}}(p+q) .
$$

Thus, by the results of Section 4.5 , the categories $\Omega^{p} M_{n}(\widehat{\mathcal{R}}(p))$ form an $\mathcal{I}$-monoid in Cat. We take the homotopy colimit (Grothendieck construction)

$$
\underset{\mathbf{p} \in \mathcal{I}}{\operatorname{hocolim}} \Omega^{m} M_{n}(\widehat{\mathcal{R}}(p))
$$

and obtain a strict monoidal category by Theorem 4.3. Using the results of Thomason [30] and Minian [20;21] we obtain the following string of weak equivalences of spaces:

$$
\begin{aligned}
B\left(\underset{\mathbf{p} \in \mathcal{I}}{\operatorname{hocolim}} \Omega^{p} M_{n}(\widehat{\mathcal{R}}(p))\right) & \simeq \underset{\mathbf{p} \in \mathcal{I}}{\operatorname{hocolim}} B\left(\Omega^{p} M_{n}(\widehat{\mathcal{R}}(p))\right) \\
& \simeq \underset{\mathbf{p} \in \mathcal{I}}{\operatorname{hocolim}} \Omega^{p} B\left(M_{n}(\widehat{\mathcal{R}}(p))\right)=\underset{\mathbf{p} \in \mathcal{I}}{\operatorname{hocolim}} \Omega^{p} M_{n}(\mathbb{E} \mathcal{R}(p)) .
\end{aligned}
$$

If we restrict to the connected components over $G L_{n}\left(\pi_{0} \mathcal{R}\right)$, we obtain a strict monoidal category $\widetilde{G L}_{n}(\mathbb{E} \mathcal{R})$ such that the classifying space $B \widetilde{G L}_{n}(\mathbb{E} \mathcal{R})$ is monoidally equivalent to the topological monoid $G L_{n}(\mathbb{E} \mathcal{R})$. We let $\widetilde{\mathcal{G L}}(\mathbb{E} \mathcal{R})$ be the 2-category with objects given by the natural numbers and categories of morphisms given by $\widetilde{G L}_{n}(\mathbb{E} \mathcal{R})$. Then we have that the category $\mathcal{G L}(\mathbb{E} \mathcal{R})$ is equivalent to $T \widetilde{\mathcal{G L}}(\mathbb{E} \mathcal{R})$. Notice moreover that block sum of matrices makes $\widetilde{\mathcal{G L}}(\mathbb{E} \mathcal{R})$ into a strict symmetric monoidal 2-category.

The second auxiliary bicategory is constructed as follows. Let $\operatorname{Mat}_{n}(\hat{\mathcal{R}}(p))$ be the category of $n \times n$ matrices over the category $\widehat{\mathcal{R}}(p)$. Since it is just $n^{2}$ copies of $\widehat{\mathcal{R}}(p)$, we get a symmetric spectrum in $C a t$. Thus, the categories $\Omega^{p} \operatorname{Mat}_{n}(\widehat{\mathcal{R}}(p))$ form an $\mathcal{I}$-category. There are also maps

$$
\mu_{p, q}: \Omega^{p} \operatorname{Mat}_{n}(\widehat{\mathcal{R}}(p)) \times \Omega^{q} \operatorname{Mat}_{n}(\widehat{\mathcal{R}}(q)) \rightarrow \Omega^{p+q} \operatorname{Mat}_{n}(\widehat{\mathcal{R}}(p+q)),
$$

given by "matrix multiplication," using the pairing $\mu_{p, q}$ as multiplication and the concatenation of loops as addition. These maps are not strictly associative. There is an 
associativity natural isomorphism between $\mu_{p, q+r} \circ\left(\mathrm{id} \times \mu_{q, r}\right)$ and $\mu_{p+q, r} \circ\left(\mu_{p, q} \times \mathrm{id}\right)$, making $\mathbf{p} \mapsto \Omega^{p} \operatorname{Mat}_{n}(\hat{\mathcal{R}}(p))$ into an $\mathcal{I}$-pseudomonoid (see Section 4.4 for definition). This in turn implies that the homotopy colimit taken as the Grothendieck construction is a monoidal category.

By taking the components over $G L_{n}\left(\pi_{0} \mathcal{R}\right)$, we obtain a monoidal category $\overline{G L}_{n}(\mathbb{E} \mathcal{R})$. We let $\overline{\mathcal{G}}(\mathbb{E} \mathcal{R})$ be the bicategory with objects given by the natural numbers, and categories of morphisms given by $\overline{G L}_{n}(\mathbb{E} \mathcal{R})$. In this case as well, block sum of matrices makes $\overline{\mathcal{G L}}(\mathbb{E} \mathcal{R})$ into a strict symmetric monoidal bicategory.

The following proposition relates the three bicategories in sight.

Theorem 4.5 There exist symmetric monoidal pseudofunctors

$$
\mathcal{G L}(\mathcal{R}) \longrightarrow \overline{\mathcal{G L}}(\mathbb{E} \mathcal{R}) \longleftarrow \widetilde{\mathcal{G L}}(\mathbb{E} \mathcal{R})
$$

Proof Since the set of objects in all three bicategories is the same, and the monoidal structure is given my block sum of matrices, it is enough to produce monoidal functors

$$
G L_{n}(\mathcal{R}) \longrightarrow \overline{G L}_{n}(\mathbb{E} \mathcal{R}) \longleftarrow \widetilde{G L}_{n}(\mathbb{E} \mathcal{R})
$$

between the categories of morphisms.

Recall from Section 4.3 that $\hat{\mathcal{R}}(0)=\mathcal{R}$. The functor $G L_{n}(\mathcal{R}) \rightarrow \overline{G L}_{n}(\mathbb{E} \mathcal{R})$ is given by the inclusion into the first term of the homotopy colimit. On the other hand, we can include $M_{n}(\hat{\mathcal{R}}(p))$ into $\operatorname{Mat}_{n}(\hat{\mathcal{R}}(p))$ as the matrices with only one nonbasepoint entry per column.

Symmetric monoidal pseudofunctors give rise to maps of $\Gamma$-bicategories. We thus obtain the following corollary.

Corollary 4.6 There is a sequence of maps of $\Gamma$-spaces

$$
\begin{aligned}
|\mathbf{N} \mathbb{W}(\mathcal{G L}(\mathcal{R}))| \longrightarrow|\mathbf{N} \mathbb{W}(\overline{\mathcal{G L}}(\mathbb{E} \mathcal{R}))| \stackrel{f}{\longleftarrow}|\mathbf{N} \mathbb{W}(\widetilde{\mathcal{G}}(\mathbb{E} \mathcal{R}))| & \simeq|\mathbf{N} \mathbb{V}(\widetilde{\mathcal{G}}(\mathbb{E} \mathcal{R}))| \\
& \simeq B T \mathbb{V}(\widetilde{\mathcal{G}}(\mathbb{E} \mathcal{R})) \\
& \simeq B \mathbb{U} \mathcal{G}(\mathbb{E} \mathcal{R}),
\end{aligned}
$$

where the map labeled $f$ is a stable equivalence.

Proof The first two maps follow from the symmetric monoidal pseudofunctors from Theorem 4.5. The map $f$ is a stable equivalence since $M_{n}(\widehat{\mathcal{R}}(m)) \cong \prod_{n} \bigvee_{n} \hat{\mathcal{R}}(m)$ and $\operatorname{Mat}_{n}(\widehat{\mathcal{R}}(m)) \cong \prod_{n^{2}} \widehat{\mathcal{R}}(m)$, and wedges and products are stably equivalent.

The rest of the equivalences come from the fact that $\mathcal{G L}(\mathbb{E} \mathcal{R}) \simeq T \widetilde{\mathcal{G L}}(\mathbb{E} \mathcal{R})$ and Corollary 2.3. 
The main theorem of this section is a consequence of Corollary 4.6 and [1, Theorem 1.1].

Theorem 4.7 There is a stable equivalence of spectra

$$
\mathbb{K}(\mathcal{R}) \stackrel{\sim}{\longrightarrow} \mathbb{K}(\mathbb{E} \mathcal{R}),
$$

which at the level of infinite loop spaces gives the map

$$
\mathcal{K}(\mathcal{R}) \underset{(3.1)}{\longrightarrow} K(\mathbb{E} \mathcal{R})
$$

Remark 4.8 In the case where $\mathcal{R}$ is a bipermutative category, the ring spectrum $\mathbb{E} \mathcal{R}$ is an $E_{\infty}$ ring spectrum, and thus $\mathbb{K}(\mathbb{E} \mathcal{R})$ is an $E_{\infty}$ ring spectrum itself. We expect that the symmetry of the multiplication in $\mathcal{R}$ will allow us to define a second monoidal structure on $\mathcal{G} \mathcal{L}(\mathcal{R})$, analogous to tensor product of modules. It is expected that following an approach similar to that of [6], we can prove that this gives an $E_{\infty}$ ring structure on $\mathbb{K}(\mathcal{R})$ compatible to the one on $\mathbb{K}(\mathbb{E} \mathcal{R})$.

\section{Constructions of $\Gamma$-bicategories}

In this section we present the construction of the special $\Gamma$-bicategory for a symmetric monoidal bicategory, and a slightly modified construction for when $\mathcal{M}$ is a 2-category. The constructions are inspired by the analogous construction for symmetric monoidal categories found in [26]. The main difference between the bicategorical and categorical cases is that certain diagrams of morphisms that were required to commute in the categorical case only commute up to 2-isomorphism in the bicategorical construction, and those 2-isomorphisms must satisfy certain coherence axioms.

\subsection{Pasting diagrams}

In the proof below we will make extensive use of pasting diagrams for bicategories. A pasting diagram is a polygonal arrangement on the plane, where the vertices correspond to objects, the directed edges correspond to 1-morphisms and the faces are usually filled with double arrows corresponding to 2-morphisms. For example, the diagram

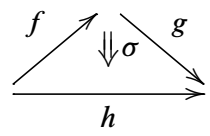

indicates that $\sigma$ is a $2-$ morphism from $g * f$ to $h$. 
We can combine pasting diagrams to depict certain compositions of 2-morphisms. For example the diagram

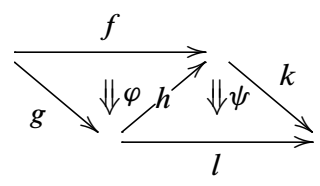

represents the 2 -morphism given by the composition

$$
k * f \stackrel{k * \varphi}{\Longrightarrow} k *(h * g) \stackrel{a}{\Longrightarrow}(k * h) * g \stackrel{\psi * g}{\Longrightarrow} l * g .
$$

We note here that the 2-morphisms are not actually composable; we need to use the associativity isomorphism. In general, for larger diagrams, the source and target of the 2-morphisms we are composing may differ by their bracketing. By the Coherence Theorem of Bicategories [14, Section 2] we know that there is a unique canonical associativity isomorphism between two bracketings, so we use these isomorphisms to connect the source and target of the 2-morphisms we are composing and hence make sense of the diagram.

Once we specify a bracketing of the outside 1-morphisms, the diagram has a unique meaning, no matter what order we use to compose the 2 -morphisms. We refer the reader to Kelly and Street [10] and Street [29, Section 3].

When we say "pasting diagram $A$ is equal to pasting diagram $B$ " we mean that with a given bracketing of the outside 1-morphisms, the given 2-morphisms that they both define are equal. Note that if this is true for a given bracketing, it is true for all bracketings.

\subsection{The proof of Theorem 2.1}

For a strict symmetric monoidal bicategory $\mathcal{M}$, we construct a $\Gamma$-bicategory as follows. The bicategory $\mathbb{W} \mathcal{M}\left(\mathbf{n}_{+}\right)$for $n \geq 0$ is given by:

(1) Objects are of the form $\left\{A_{S}, a_{S, T}\right\}_{S, T}$, where $S$ runs over all the subsets of $\mathbf{n}_{+}$that do not contain the basepoint $0 ;(S, T)$ runs over all pairs of such subsets such that $S \cap T=\varnothing ; A_{S} \in \mathrm{Ob} \mathcal{M}$ and $a_{S, T}: A_{S \cup T} \rightarrow A_{S} \boxplus A_{T}$ is a 1 -equivalence, that is a 1 -morphism that is invertible up to isomorphism. We require further 
(a) $A_{\varnothing}=0$;

(b) $a_{\varnothing, S}=I_{A_{S}}=a_{S, \varnothing}$;

(c) for every triple $(S, T, U)$ of subsets such that $S \cap T=S \cap U=T \cap U=\varnothing$, the diagram

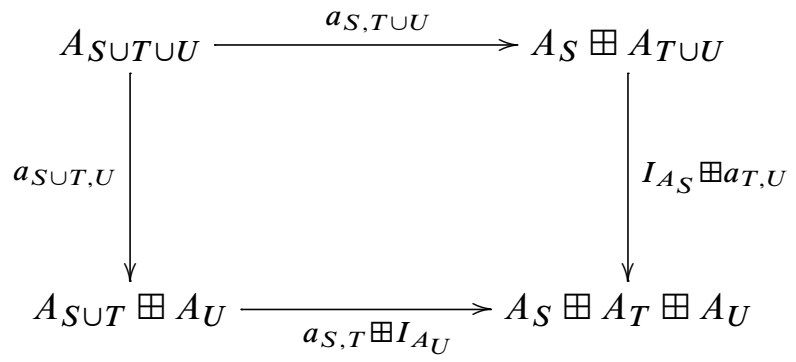

strictly commutes;

(d) for every pair of subsets $(S, T)$, the diagram

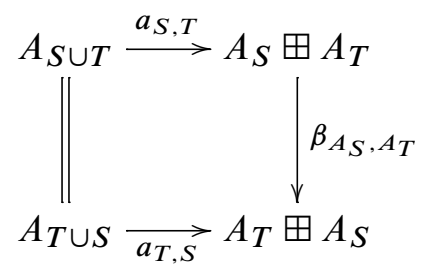

strictly commutes.

(2) A 1-morphism $\left\{A_{S}, a_{S, T}\right\} \rightarrow\left\{A_{S}^{\prime}, a_{S, T}^{\prime}\right\}$ is given by a system $\left\{f_{S}, \phi_{S, T}\right\}_{S, T}$, where $S, T$ are as above; $f_{S}: A_{S} \rightarrow A_{S}^{\prime}$ is a 1-morphism in $\mathcal{M}$ and $\phi_{S, T}$ is a 2-isomorphism:

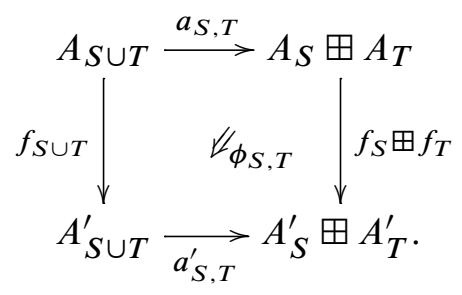

We require:

(a) $\phi_{\varnothing, S}: f_{S} * I_{A_{S}}=f_{S} \Rightarrow f_{S}=I_{A_{S}} * f_{S}$ is the identity 2-morphism and similarly for $\phi_{S, \varnothing}$; 
(b) for every pairwise disjoint $S, T, U$ the following equation of pasting diagrams holds:
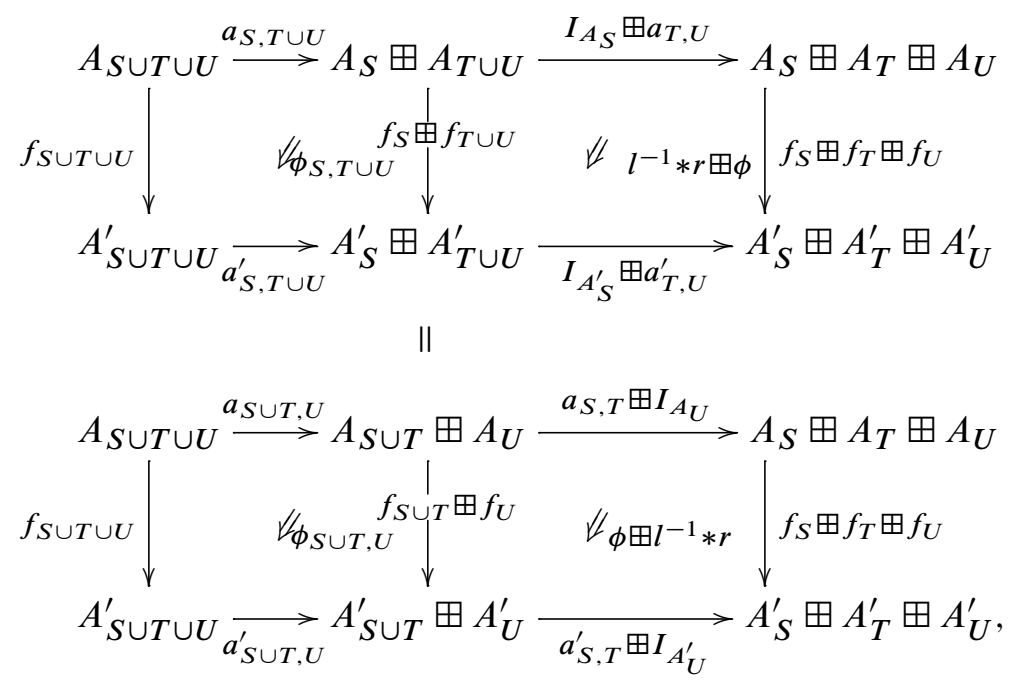

where $r, l$ denote the coherent identity isomorphisms in $\mathcal{M}$, that is $l_{f}: I_{B} *$ $f \rightarrow f$ and $r_{f}: f * I_{A} \rightarrow f$;

(c) for every $S, T$ the following equation holds:

$$
A_{S \cup T} \stackrel{a_{S, T}}{\longrightarrow} A_{S} \boxplus A_{T} \stackrel{\beta}{\longrightarrow} A_{T} \boxplus A_{S} \quad A_{T \cup S} \stackrel{a_{T, S}}{\longrightarrow} A_{T} \boxplus A_{S}
$$

(5.5) $\quad f_{S \cup T} \downarrow \quad \Downarrow_{\phi_{S, T}} f_{V}^{\mid} \boxplus f_{T}=\quad f_{T} \boxplus f_{S}=f_{T \cup S} \downarrow \quad \forall \phi_{T, S} \downarrow f_{T} \boxplus f_{S}$

$$
A_{S \cup T}^{\prime} \underset{a_{S, T}^{\prime}}{\longrightarrow} A_{S}^{\prime} \boxplus A_{T}^{\prime} \underset{\beta}{\longrightarrow} A_{T}^{\prime} \boxplus A_{S}^{\prime} \quad A_{T \cup S}^{\prime} \underset{a_{T, S}^{\prime}}{\longrightarrow} A_{T}^{\prime} \boxplus A_{S}^{\prime} .
$$

(3) Given 1-morphisms

$$
\left\{f_{S}, \phi_{S, T}\right\},\left\{g_{S}, \gamma_{S, T}\right\}:\left\{A_{S}, a_{S, T}\right\} \rightarrow\left\{A_{S}^{\prime}, a_{S, T}^{\prime}\right\},
$$

a 2-morphism between them is given by a system $\left\{\psi_{S}\right\}$ of 2 -morphisms in $\mathcal{M}$, $\psi_{S}: f_{S} \Rightarrow g_{S}$, such that for all $S, T$ as above the following equation holds:

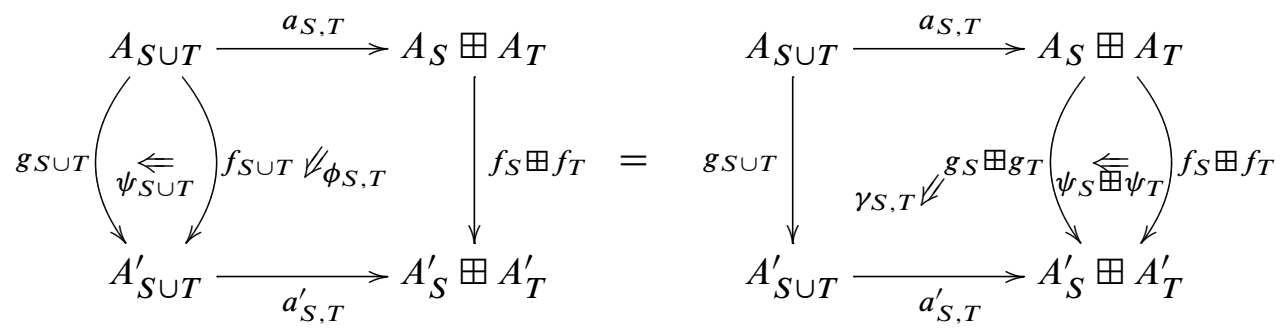


Remark 5.6 We note that the symmetric monoidal structure being strict allows us to use this simple construction. The reader will remark that in the definition of the objects we require certain diagrams to commute strictly, as opposed to having a $2-$ isomorphism relating the two sides. This can be done because the braiding $\beta$ satisfies the commutativity of the diagrams of Definition 1.3 on the nose. This cannot be done for the definition of the 1-morphisms, and this is where the construction here differs from that of [26]; in order to get a well-defined bicategory we cannot require the diagram in (5.3) to commute, instead we must have the isomorphism $\phi_{S, T}$ as part of the data of the 1-morphism.

We now need to show that these data indeed define a bicategory. We will first show that given objects $\left\{A_{S}, a_{S, T}\right\},\left\{A_{S}^{\prime}, a_{S, T}^{\prime}\right\}$, the 1-morphisms and 2-morphisms form a category $\mathbb{W} \mathcal{M}\left(\mathbf{n}_{+}\right)\left(\left\{A_{S}, a_{S, T}\right\},\left\{A_{S}^{\prime}, a_{S, T}^{\prime}\right\}\right)$.

Given two 2-morphisms $\left\{\psi_{S}\right\},\left\{\psi_{S}^{\prime}\right\}$, vertical composition is defined componentwise. We show that this composition satisfies Equation (5.7). Indeed, we see that, as wanted, for all $S, T$,
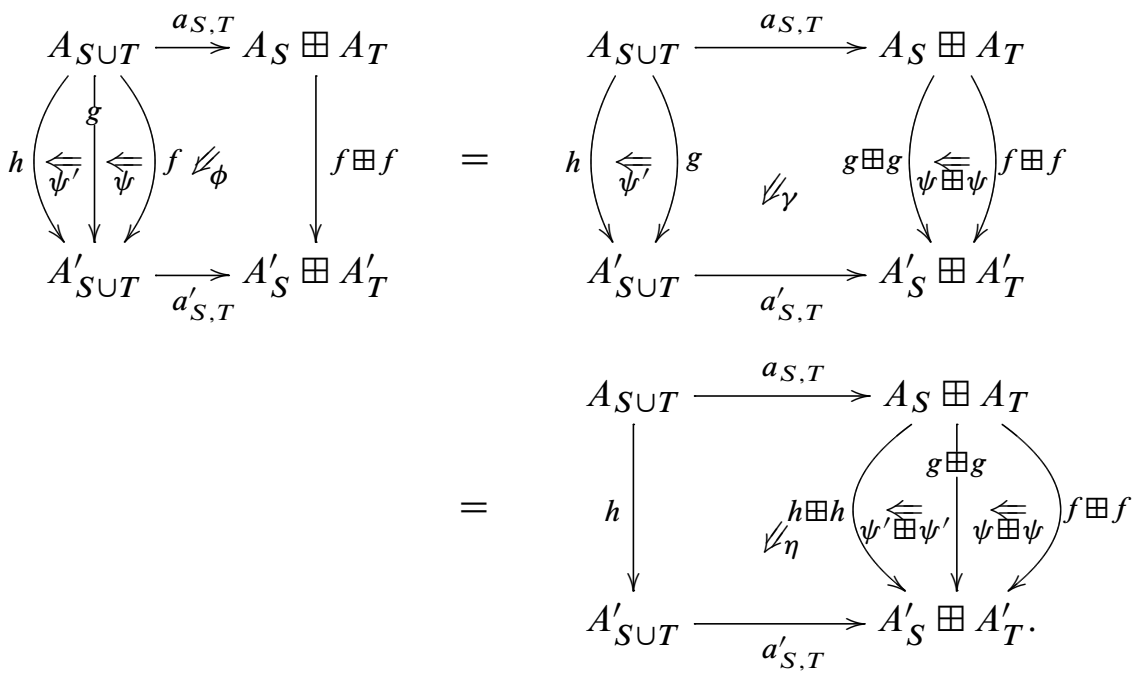

We also note that $\left\{\operatorname{id}_{f_{S}}\right\}$ is a well-defined automorphism for $\left\{f_{S}, \phi_{S, T}\right\}$ and it is the identity of the componentwise composition.

The composition functor $*$ is given by

$$
\begin{aligned}
\left(\left\{g_{S}, \gamma_{S, T}\right\},\left\{f_{S}, \phi_{S, T}\right\}\right) & \mapsto\left\{g_{S} * f_{S},(\gamma \diamond \phi)_{S, T}\right\}, \\
\left(\left\{\psi_{S}^{\prime}\right\},\left\{\psi_{S}\right\}\right) & \mapsto\left\{\psi_{S}^{\prime} * \psi_{S}\right\},
\end{aligned}
$$


where the 2 -morphism $(\gamma \diamond \phi)_{S, T}$ is defined by the pasting diagram

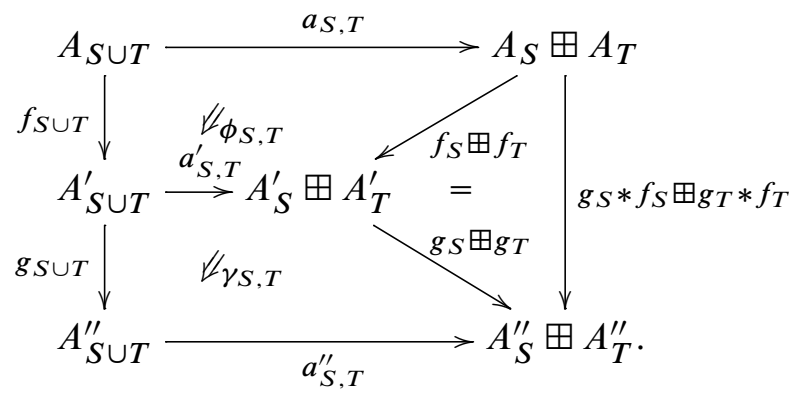

Showing that $\left\{g_{S} * f_{S},(\gamma \diamond \phi)_{S, T}\right\}$ is a well-defined 1-morphism (that is, it satisfies Equations (5.4) and (5.5)) can be done again using pasting diagrams, and the fact that both $\left\{f_{S}, \phi_{S, T}\right\}$ and $\left\{g_{S}, \gamma_{S, T}\right\}$ satisfy those same equations. Analogously we can show that $\left\{\psi_{S}^{\prime} * \psi_{S}\right\}$ is a well-defined 2-morphism.

The natural associativity isomorphism in this bicategory is given by the componentwise associativity isomorphisms in $\mathcal{M}$. More precisely, given composable 1-morphisms $\left\{f_{S}, \phi_{S, T}\right\},\left\{g_{S}, \gamma_{S, T}\right\}$, and $\left\{h_{S}, \eta_{S, T}\right\}$, we define the 2-morphism $\left\{\alpha_{S}\right\}$, where

$$
\alpha_{S}: h_{S} *\left(g_{S} * f_{S}\right) \Rightarrow\left(h_{S} * g_{S}\right) * f_{S}
$$

is the associativity isomorphism in $\mathcal{M}$.

The fact that

$$
\left\{\left(h_{S} * g_{S}\right) * f_{S},((\eta \diamond \gamma) \diamond \phi)_{S, T}\right\} \Rightarrow\left\{h_{S} *\left(g_{S} * f_{S}\right),(\eta \diamond(\gamma \diamond \phi))_{S, T}\right\}
$$

is an allowed $2-$ morphism in $\mathbb{W} \mathcal{M}\left(\mathbf{n}_{+}\right)$will follow from the uniqueness of pasting diagrams and the functoriality of $\boxplus$. Naturality and the pentagonal axiom follow from those in $\mathcal{M}$.

Given an object $\left\{A_{S}, a_{S, T}\right\}$, the identity 1 -morphism is given by $\left\{I_{A_{S}}, \iota_{S, T}\right\}$, where $\iota$ is the appropriate coherent 2-isomorphism obtained by the composition of instances of the 2 -morphism $r^{-1} * l$. It is clear that this is an allowed 1 -morphism in $\mathbb{W} \mathcal{M}\left(\mathbf{n}_{+}\right)$ and that it is a weak identity, with right and left identity 2 -isomorphisms given by $\left\{r_{f_{S}}\right\},\left\{l_{f_{S}}\right\}$. We conclude thus that $\mathbb{W} \mathcal{M}\left(\mathbf{n}_{+}\right)$is indeed a bicategory.

We now need to prove that this construction extends to a functor $\mathbb{W} \mathcal{M}: \mathcal{F} \rightarrow$ Bicat $_{*}$. Given a morphism $\theta: \mathbf{n}_{+} \rightarrow \mathbf{m}_{+}$in $\mathcal{F}$ we define a pseudofunctor

$$
\theta_{*}: \mathbb{W} \mathcal{M}\left(\mathbf{n}_{+}\right) \rightarrow \mathbb{W} \mathcal{M}\left(\mathbf{m}_{+}\right)
$$


as follows:

$$
\begin{aligned}
\left\{A_{S}, a_{S, T}\right\} & \longmapsto\left\{A_{U}^{\theta}, a_{U, V}^{\theta}\right\}=\left\{A_{\theta^{-1}(U)}, a_{\theta^{-1}(U), \theta^{-1}(V)}\right\}, \\
\left\{f_{S}, \phi_{S, T}\right\} & \longmapsto\left\{f_{U}^{\theta}, \phi_{U, V}^{\theta}\right\}=\left\{f_{\theta^{-1}(U)}, \phi_{\theta^{-1}(U), \theta^{-1}(V)}\right\}, \\
\left\{\psi_{S}\right\} & \longmapsto\left\{\psi_{U}^{\theta}\right\}=\left\{\psi_{\theta^{-1}(U)}\right\},
\end{aligned}
$$

where $U, V$ range over disjoint subsets of $\mathbf{m}_{+}$that do not contain the basepoint. Since $\theta$ is basepoint preserving, $\theta^{-1}(U)$ does not contain the basepoint and it is an allowed indexing subset of $\mathbf{n}_{+}$. Also, since $U$ and $V$ are disjoint, their preimages under $\theta$ are also disjoint.

This assignment commutes strictly with all the compositions and identities in $\mathbb{W} \mathcal{M}\left(\mathbf{n}_{+}\right)$ and $\mathbb{W} \mathcal{M}\left(\mathbf{m}_{+}\right)$, giving a pseudofunctor between these bicategories.

It is clear from the construction that $\mathbb{W} \mathcal{M}\left(\mathbf{1}_{+}\right)$is isomorphic to $\mathcal{M}$.

We will end the proof by showing that for every $n \geq 0$, the pseudofunctor

$$
p_{n}: \mathbb{W} \mathcal{M}\left(\mathbf{n}_{+}\right) \rightarrow \mathcal{M}^{\times n}
$$

is an equivalence of bicategories (Definition 1.5). This will show that the $\Gamma$-bicategory is special. For ease of notation we will denote the subset $\{i\} \in \mathbf{n}_{+}$as $i$. The pseudofunctor $p_{n}$ takes

$$
\begin{aligned}
\left\{A_{S}, a_{S, T}\right\} & \longmapsto\left\{A_{i}\right\}_{i=1}^{n}, \\
\left\{f_{S}, \phi_{S, T}\right\} & \longmapsto\left\{f_{i}\right\}_{i=1}^{n}, \\
\left\{\psi_{S}\right\} & \longmapsto\left\{\psi_{i}\right\}_{i=1}^{n} .
\end{aligned}
$$

We will define an inverse pseudofunctor $i_{n}: \mathcal{M}^{\times n} \rightarrow \mathbb{W} \mathcal{M}\left(\mathbf{n}_{+}\right):$

$$
\begin{aligned}
&\left\{A_{i}\right\}_{i=1}^{n} \longmapsto\left\{\bigoplus_{i \in S} A_{i}, e_{S, T}\right\}, \\
&\left\{f_{i}\right\}_{i=1}^{n} \longmapsto\left\{\bigoplus_{i \in S} f_{i}, \mathrm{id}\right\}, \\
&\left\{\psi_{i}\right\}_{i=1}^{n} \longmapsto\left\{\bigoplus_{i \in S} \psi_{i}\right\} .
\end{aligned}
$$

Here, $\boxplus_{i \in S}$ denotes the iterated monoidal operation $\boxplus$ with the usual order of the elements in $S \subset \mathbf{n}_{+}$. Recall that $\boxplus$ is strictly associative.

The 1-morphism

$$
e_{S, T}: \boxplus \bigoplus_{i \in S \cup T} A_{i} \longrightarrow \boxplus_{i \in S} A_{i} \boxplus \bigoplus_{i \in T} A_{i}
$$


is the unique composition of instances of the braiding $\beta$ that reorders the summands. It is clear that $\left\{\boxplus_{i \in S} A_{i}, e_{S, T}\right\}$ satisfies Equations (5.1) and (5.2).

We also have that $\left(\boxplus_{i \in S} f_{i} \boxplus \boxplus_{i \in T} f_{i}\right) * e_{S, T}=e_{S, T} *\left(\boxplus_{i \in S \cup T} f_{i}\right)$, thus we can choose the 2-isomorphism to be the identity. The collection $\left\{\boxplus_{i \in S} f_{i}\right.$, id satisfies automatically Equations (5.4) and (5.5). It is also automatic that for any $\left\{\psi_{i}\right\}:\left\{f_{i}\right\} \Rightarrow\left\{g_{i}\right\}$, we get that $\left\{\boxplus_{i \in S} \psi_{i}\right\}$ is an allowed 2-morphism between $\left\{\boxplus_{i \in S} f_{i}, \mathrm{id}\right\}$ and $\left\{\boxplus_{i \in S} g_{i}, \mathrm{id}\right\}$.

This assignment gives a strict functor since

$$
\begin{aligned}
i_{n}\left(\left\{g_{i} * f_{i}\right\}\right) & =\left\{\bigoplus_{i \in S}\left(g_{i} * f_{i}\right), \mathrm{id}\right\} \\
& =\left\{\left(\bigoplus_{i \in S} g_{i}\right) *\left(\bigoplus_{i \in S} f_{i}\right), \mathrm{id} \diamond \mathrm{id}\right\}=i_{n}\left(\left\{g_{i}\right\}\right) * i_{n}\left(\left\{f_{i}\right\}\right), \\
i_{n}\left(\left\{I_{A_{i}}\right\}\right) & =\left\{\bigoplus_{i \in S} I_{A_{i}}, \mathrm{id}\right\}=\left\{I_{\boxplus_{i \in S} A_{i}}, \mathrm{id}\right\}=\operatorname{id}_{i_{n}\left(\left\{A_{i}\right\}\right) .}
\end{aligned}
$$

Clearly $p_{n} \circ i_{n}=\mathrm{id}_{\mathcal{M}^{\times n}}$. We now construct a natural equivalence

$$
\xi: \operatorname{id}_{\mathbb{W} \mathcal{M}\left(\mathbf{n}_{+}\right)} \rightarrow i_{n} \circ p_{n} .
$$

Recall that a transformation is a natural equivalence if and only if the 1-morphism corresponding to each object is a 1 -equivalence.

Hence, to construct the natural equivalence $\xi$, we need a 1 -equivalence

$$
\xi_{\left\{A_{S}, a_{S, T}\right\}}:\left\{A_{S}, a_{S, T}\right\} \rightarrow\left\{\bigoplus_{i \in S} A_{i}, e_{S, T}\right\}
$$

for every object $\left\{A_{S}, a_{S, T}\right\}$ in $\mathbb{W} \mathcal{M}\left(\mathbf{n}_{+}\right)$.

Given the subset $S$, we define $a^{S}$ inductively as the composition

$$
A_{S} \stackrel{a_{j, S-j}}{\longrightarrow} A_{j} \boxplus A_{S-j} \stackrel{\mathrm{id}_{A_{j}} \boxplus a^{S-j}}{\longrightarrow} A_{j} \boxplus \underset{i \in S-j}{\boxplus_{i}} A_{i}=\bigoplus_{i \in S} A_{i},
$$

where $j$ is the smallest element in $S$.

Note that by conditions (5.1) and (5.2) on the $a_{S, T}$, the two compositions in the diagram below differ by a specified associativity 2 -isomorphism:

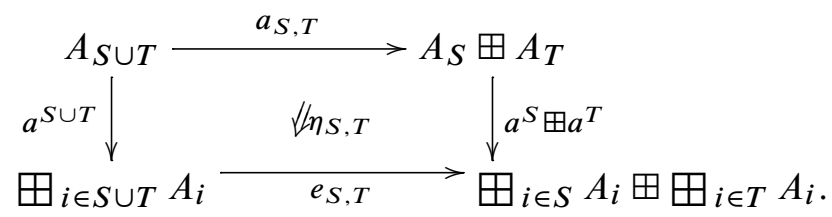


Since associativity isomorphisms are unique, $\left\{a^{S}, \eta_{S, T}\right\}$ is a well-defined 1-morphism in $\mathbb{W} \mathcal{M}\left(\mathbf{n}_{+}\right)$. This will be the corresponding 1-morphism of the transformation $\xi$.

To complete the data of the transformation, we need to provide a natural isomorphism $7 \xi^{2}$ for every pair of objects $\left\{A_{S}, a_{S, T}\right\},\left\{A_{S}^{\prime}, a_{S, T}^{\prime}\right\}$ in $\mathbb{W} \mathcal{M}\left(\mathbf{n}_{+}\right)$, which on the component $\left\{f_{S}, \phi_{S, T}\right\}$ is given by a 2 -morphism

$$
\xi^{2}\left(\left\{f_{S}, \phi_{S, T}\right\}\right):\left\{\bigoplus_{i \in S} f_{i}, \mathrm{id}\right\} *\left\{a^{S}, \eta_{S, T}\right\} \Rightarrow\left\{a^{\prime S}, \eta_{S, T}^{\prime}\right\} *\left\{f_{S}, \phi_{S, T}\right\} .
$$

Given $S$, we define a 2-isomorphism in $\mathcal{M}, \phi^{S}$ : (田 $\left.{ }_{i \in S} f_{i}\right) * a^{S} \Rightarrow a^{\prime S} * f_{S}$, inductively as the pasting diagram

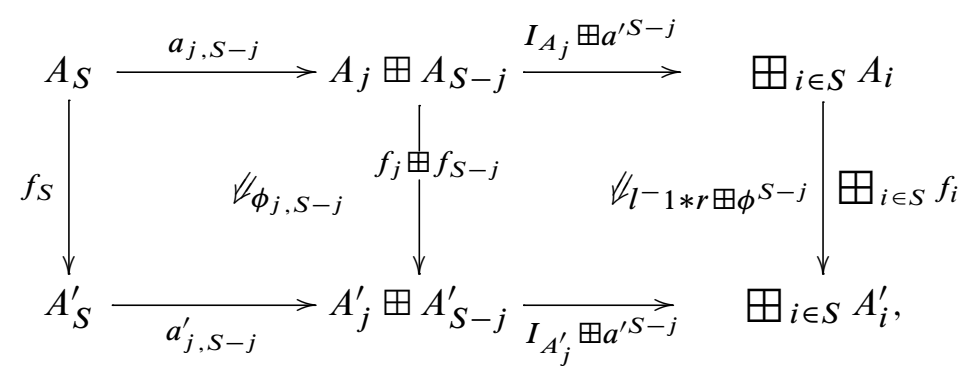

where $j$ is the smallest element in $S$. We need to show that $\left\{\phi^{S}\right\}_{S}$ gives a 2-morphism in $\mathbb{W} \mathcal{M}\left(\mathbf{n}_{+}\right)$, that is, that it satisfies Equation (5.7). This is done by induction on $|S \cup T|$ using pasting diagrams. We let $\xi^{2}\left(\left\{f_{S}, \phi_{S, T}\right\}\right)=\left\{\phi^{S}\right\}$.

To show the naturality of $\xi^{2}$, we need to show that
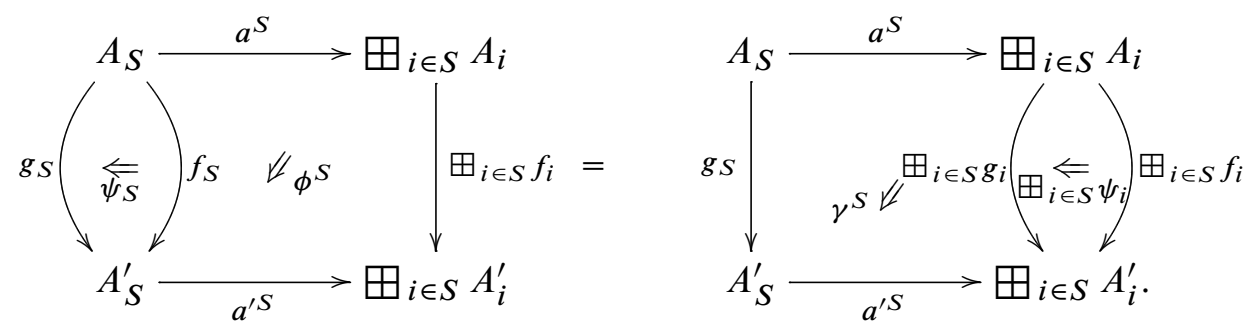

This follows by induction on $|S|$, using the inductive definition of $\phi^{S}$ and Equation (5.7). Since $\phi^{S}$ is invertible, we get a natural isomorphism as wanted. 
For the first and second axioms of a transformation [29, page 568] we need to show:
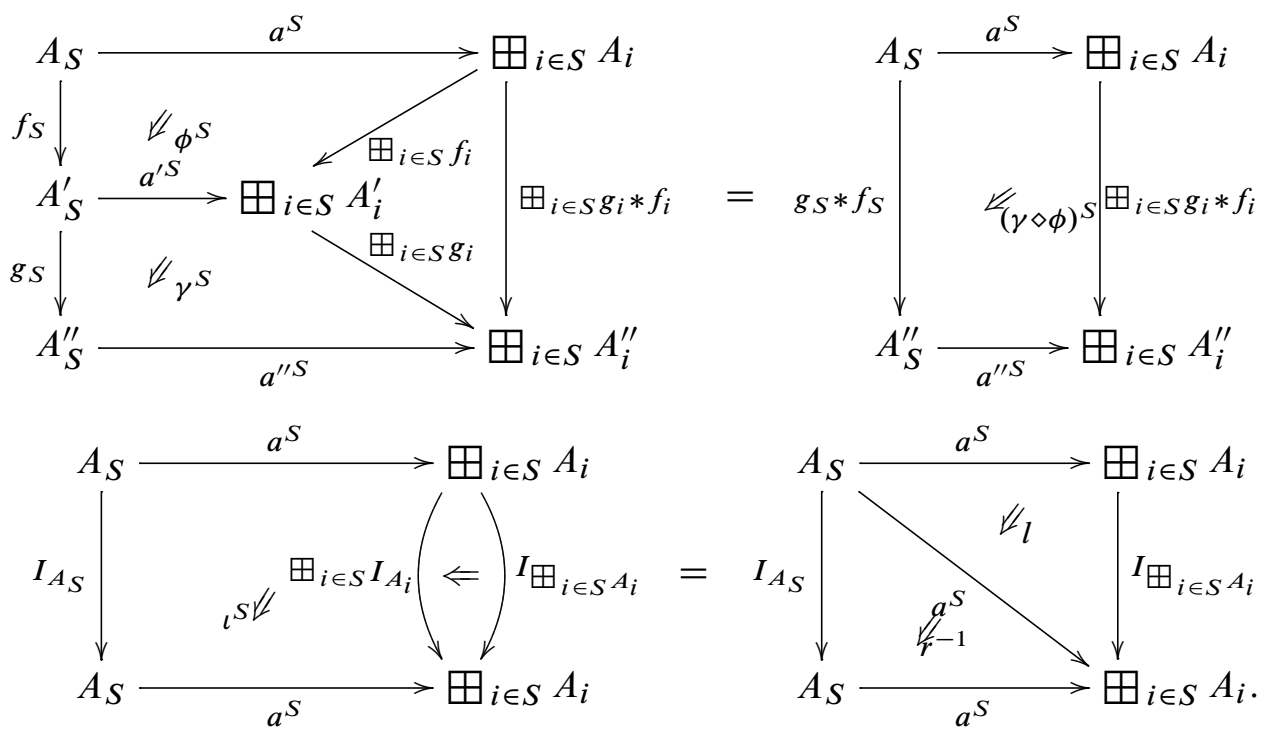

The first one is straightforward using induction on $|S|$ and the definition of $\gamma \diamond \phi$. The second one holds again by induction on $|S|$, the fact that $\iota$ is a composition of instances of $r$ and $l$ which satisfy coherence conditions.

Hence we have a natural equivalence between $\operatorname{id}_{\mathbb{W} \mathcal{M}\left(\mathbf{n}_{+}\right)}$and $i_{n} \circ p_{n}$. We conclude that the bicategories $\mathbb{W} \mathcal{M}\left(\mathbf{n}_{+}\right)$and $\mathcal{M}^{\times n}$ are equivalent, making $\mathbb{W} \mathcal{M}$ into a special $\Gamma$-bicategory.

\subsection{The proof of Theorem 2.2}

We now turn to the case of $2-$ categories. We note that if $\mathcal{M}$ is a strict symmetric monoidal 2-category, with the braiding $\beta$ a strict natural isomorphism, then we can construct a $\Gamma-(2-$ category $)$ in a similar but simpler way.

(1) Objects of $(\mathbb{V} \mathcal{M})\left(\mathbf{n}_{+}\right)$are the same as objects of $(\mathbb{W} \mathcal{M})\left(\mathbf{n}_{+}\right)$.

(2) A 1-morphism between $\left\{A_{S}, a_{S, T}\right\}$ and $\left\{A_{S}^{\prime}, a_{S, T}^{\prime}\right\}$ is given by a system $\left\{f_{S}\right\}$, where $f_{S}: A_{S} \rightarrow A_{S}^{\prime}$ is a 1 -morphism in $\mathcal{M}$. We require that for all $S$ and $T$ the following diagram commutes strictly:

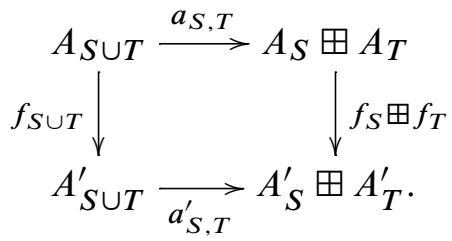


(3) Given 1-morphisms $\left\{f_{S}\right\},\left\{g_{S},\right\}:\left\{A_{S}, a_{S, T}\right\} \rightarrow\left\{A_{S}^{\prime}, a_{S, T}^{\prime}\right\}$, a 2-morphism between them is given by a system $\left\{\psi_{S}\right\}$ of 2 -morphisms in $\mathcal{M}, \psi_{S}: f_{S} \Rightarrow g_{S}$, such that for all $S, T$ as above the following equation holds:

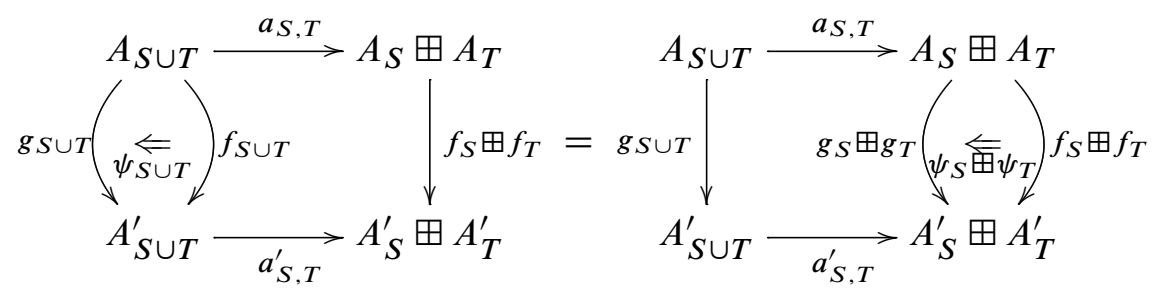

In this case, vertical and horizontal composition is defined componentwise, and it is easy to check that this is a well defined 2-category. Using the same methods as above, we can extend this to be a $\Gamma-(2-$ category $) \mathbb{V} \mathcal{M}$, and prove that is it special. There is an inclusion of $\Gamma-(2-$ categories $) ~ j: \mathbb{V} \mathcal{M} \rightarrow \mathbb{W} \mathcal{M}$ that makes the following diagram commute:

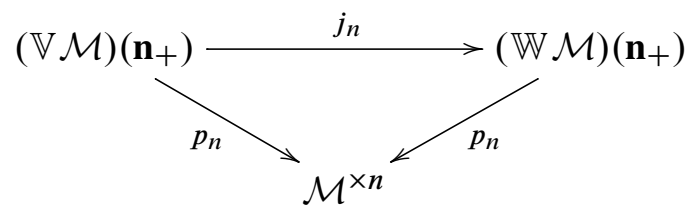

Thus $j$ is a levelwise equivalence.

Consider the topologically enriched category $T \mathcal{M}$. Since $\mathcal{M}$ is strict symmetric monoidal, $T \mathcal{M}$ is a permutative category. Thus we can construct a special $\Gamma$-category $\mathbb{U} T \mathcal{M}$ as in [26]. We now compare this $\Gamma$-category with $T \mathbb{V} \mathcal{M}$.

Note that the objects in the categories $T(\mathbb{V} \mathcal{M})\left(\mathbf{n}_{+}\right)$and $\mathbb{U} T \mathcal{M}\left(\mathbf{n}_{+}\right)$are the same. Given objects $A=\left\{A_{S}, a_{S, T}\right\}$ and $A^{\prime}=\left\{A_{S}^{\prime}, a_{S, T}^{\prime}\right\}$, the spaces of morphisms correspond to the same subspace of $B \prod_{S} \mathcal{M}\left(A_{S}, A_{S}^{\prime}\right)$ :

$$
\begin{aligned}
& B(\mathbb{V} \mathcal{M})\left(\mathbf{n}_{+}\right)\left(A, A^{\prime}\right) \subseteq B \prod_{S} \mathcal{M}\left(A_{S}, A_{S}^{\prime}\right) \\
& \mathbb{U} T \mathcal{M}\left(\mathbf{n}_{+}\right)\left(A, A^{\prime}\right) \subseteq \prod_{S} B \mathcal{M}\left(A_{S}, A_{S}^{\prime}\right) .
\end{aligned}
$$

Thus we have maps of $\Gamma$-categories

$$
\mathbb{U} T \mathcal{M} \stackrel{\cong}{\rightarrow} T \mathbb{V} \mathcal{M} \stackrel{\simeq}{\rightarrow} T \mathbb{W} \mathcal{M},
$$

where the first map is a levelwise isomorphism and the second is a levelwise equivalence. 


\section{Appendix A Classifying spaces of bicategories}

Categories are closely related to spaces through the classifying space construction. To every category we can assign a topological space, with the property that functors are sent to continuous maps, and natural transformations are sent to homotopies.

The same can be done with bicategories. In fact, there are many distinct constructions of the classifying space of a bicategory [4]. All these constructions give equivalent spaces. Here we describe the version of the two versions of the classifying space that were used in the preceding sections.

Lack and Paoli [11] introduce a version of a nerve of nonenriched bicategories that gives rise to a simplicial object in the category of small (nonenriched) categories. The construction can be extended to the enriched case, giving a simplicial object in Cat, where Cat is the category of small categories enriched over topological spaces. This construction is closely related to the bar construction for monoidal categories defined in [2], as we point out. This nerve is called 2-nerve in [11] and Segal nerve in [4].

Definition A.1 Let $\mathcal{C}$ be a bicategory. The $2-$ nerve $\mathbf{N} \mathcal{C}$ is the simplicial object in Cat given by normal homomorphisms, that is,

$$
\mathbf{N}_{n} \mathcal{C}=\underline{\operatorname{NorHom}}([n], \mathcal{C}),
$$

where the objects are normal pseudofunctors and the morphisms are icons.

Normal pseudofunctors are those for which the identity natural isomorphism is the identity. An icon (Identity Component Oplax Natural transformation) is an oplax natural transformation (see [29, page 568]) such that the map $\eta_{A}: \mathcal{F} A \rightarrow \mathcal{G} A$ is the identity, so in particular, we require that $\mathcal{F} A=\mathcal{G} A$. We now unravel Definition A.1.

An object of $\mathbf{N}_{n} \mathcal{C}$ is given then by a collection of diagrams

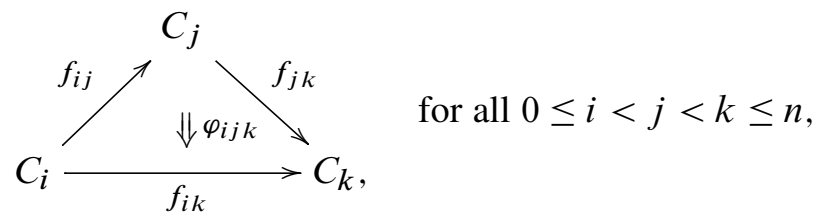

where $\varphi_{i j k}$ is an invertible $2-$ morphism. This collection must satisfy the following 
coherence condition for all $0 \leq i<j<k<l \leq n$ :
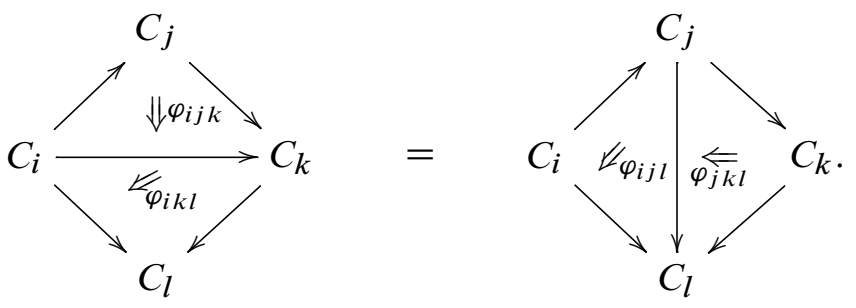

Given objects $\left\{C_{i}, f_{i j}, \varphi_{i j k}\right\}$ and $\left\{C_{i}, f_{i j}^{\prime}, \varphi_{i j k}^{\prime}\right\}$ (note that the collections of objects are equal), a morphism between them is given by a collection of 2-morphisms $\eta_{i j}: f_{i j} \Rightarrow f_{i j}^{\prime}$ for $i \leq j$, such that some coherence conditions [4, Equation (44)] are satisfied.

Remark A.2 We note that the bar construction for monoidal categories of [2] is equal to the 2-nerve. More precisely, if $\mathcal{M}$ is a monoidal category, then the simplicial category B. $\mathcal{M}$ of [2] is equal to $\mathbf{N} \Sigma \mathcal{M}$. Furthermore (see [4, Remark 3.3]), since $\mathbf{N}_{p} \Sigma \mathcal{M} \simeq \mathcal{M}^{p}$, the geometric realization $|\mathbf{N} \Sigma \mathcal{M}|$ gives a model for the delooping of the $A_{\infty}$-space $B \mathcal{M}$, that is, $B B \mathcal{M} \simeq|N \Sigma \mathcal{M}|$.

The 2-nerve is functorial with respect to normal pseudofunctors. It is the case that any pseudofunctor can be normalized [11, Proposition 5.2].

It is clear from the definition that the 2-nerve preserves products.

Definition A.3 Let $\mathcal{C}$ be a bicategory. The classifying space of $\mathcal{C}$ is the realization $|\mathbf{N C}|$.

Let $\mathcal{F}, \mathcal{G}: \mathcal{C} \rightarrow \mathcal{D}$ be pseudofunctors of bicategories, and $\eta: \mathcal{F} \rightarrow \mathcal{G}$ a transformation. As pointed out in the proof of [4, Proposition 7.1], these data gives rise to a pseudofunctor

$$
\mathcal{H}: \mathcal{C} \times \mathbf{1} \rightarrow \mathcal{D}
$$

that restricts to $\mathcal{F}$ and $\mathcal{G}$ at 0 and 1 . This pseudofunctor can be normalized, yielding the following result:

Proposition A.4 A transformation between pseudofunctors $\mathcal{F}, \mathcal{G}: \mathcal{C} \rightarrow \mathcal{D}$ gives rise to a homotopy between the maps

$$
|\mathbf{N} \mathcal{F}|,|\mathbf{N} \mathcal{G}|:|\mathbf{N} \mathcal{C}| \rightarrow|\mathbf{N} \mathcal{D}|
$$


An alternative construction for the classifying space of a bicategory $\mathcal{C}$ is described in [4]. It is the classifying space of the pseudosimplicial category whose $n$ simplices are given by

$$
\coprod_{C_{0}, \ldots, C_{n}} \mathcal{C}\left(C_{0}, C_{1}\right) \times \cdots \times \mathcal{C}\left(C_{n-1}, C_{n}\right) .
$$

As pointed out in [4, Remark 3.2], if $\mathcal{C}$ is a 2 -category, then $B \mathcal{C}$ can be constructed by first taking the classifying space of the categories of morphisms, to obtain $T \mathcal{C}$, which is a category enriched over topological spaces, and then taking $B T C$.

By [4, Theorem 6.4], there is an equivalence of spaces $B T C \rightarrow|\mathbf{N C}|$.

\section{References}

[1] N A Baas, B I Dundas, B Richter, J Rognes, Stable bundles over rig categories, J. Topol. 4 (2011) 623-640 MR2832571

[2] N A Baas, B I Dundas, J Rognes, Two-vector bundles and forms of elliptic cohomology, from: "Topology, geometry and quantum field theory", (U Tillmann, editor), London Math. Soc. Lecture Note Ser. 308, Cambridge Univ. Press (2004) 18-45 MR2079370

[3] J Bénabou, Introduction to bicategories, from: "Reports of the Midwest Category Seminar”, Springer, Berlin (1967) 1-77 MR0220789

[4] P Carrasco, A M Cegarra, A R Garzón, Nerves and classifying spaces for bicategories, Algebr. Geom. Topol. 10 (2010) 219-274 MR2602835

[5] B Day, R Street, Monoidal bicategories and Hopf algebroids, Adv. Math. 129 (1997) 99-157 MR1458415

[6] A D Elmendorf, M A Mandell, Rings, modules, and algebras in infinite loop space theory, Adv. Math. 205 (2006) 163-228 MR2254311

[7] R Gordon, A J Power, R Street, Coherence for tricategories, Mem. Amer. Math. Soc. 117, no. 558, Amer. Math. Soc. (1995) MR1261589

[8] B J Guillou, Strictification of categories weakly enriched in symmetric monoidal categories, Theory Appl. Categ. 24 (2010) No. 20, 564-579 MR2770075

[9] M M Kapranov, V A Voevodsky, 2-categories and Zamolodchikov tetrahedra equations, from: "Algebraic groups and their generalizations: quantum and infinitedimensional methods (University Park, PA, 1991)”, (W J Haboush, B J Parshall, editors), Proc. Sympos. Pure Math. 56, Amer. Math. Soc. (1994) 177-259 MR1278735

[10] G M Kelly, R Street, Review of the elements of 2-categories, from: "Category Seminar (Proc. Sem., Sydney, 1972/1973)", (G M Kelly, editor), Lecture Notes in Math. 420, Springer, Berlin (1974) 75-103 MR0357542 
[11] S Lack, S Paoli, 2-nerves for bicategories, K-Theory 38 (2008) 153-175 MR2366560

[12] ML Laplaza, Coherence for distributivity, from: "Coherence in categories", (S Mac Lane, editor), Lecture Notes in Math. 281, Springer, Berlin (1972) 29-65 MR0335598

[13] T Leinster, Higher operads, higher categories, London Math. Soc. Lecture Note Series 298, Cambridge Univ. Press (2004) MR2094071

[14] S Mac Lane, R Paré, Coherence for bicategories and indexed categories, J. Pure Appl. Algebra 37 (1985) 59-80 MR794793

[15] MA Mandell, An inverse K-theory functor, Doc. Math. 15 (2010) 765-791 MR2735988

[16] J P May, $E_{\infty}$ ring spaces and $E_{\infty}$ ring spectra, Lecture Notes in Math. 577, Springer, Berlin (1977) MR0494077 With contributions by F Quinn, N Ray, and J Tornehave

[17] J P May, The spectra associated to permutative categories, Topology 17 (1978) 225228 MR508886

[18] J P May, The construction of $E_{\infty}$ ring spaces from bipermutative categories, from: "New topological contexts for Galois theory and algebraic geometry (BIRS 2008)", (A Baker, B Richter, editors), Geom. Topol. Monogr. 16, Geom. Topol. Publ., Coventry (2009) 283-330 MR2544392

[19] P McCrudden, Balanced coalgebroids, Theory Appl. Categ. 7 (2000) 71-147 MR1764504

[20] E G Minian, Loop and suspension functors for small categories and stable homotopy groups, Appl. Categ. Structures 11 (2003) 207-218 MR1978439

[21] E G Minian, Spectra of small categories and infinite loop space machines, $K$-Theory 37 (2006) 249-261 MR2273459

[22] D Quillen, Higher algebraic $K$-theory. I, from: "Algebraic $K$-theory, I: Higher $K-$ theories (Proc. Conf., Battelle Memorial Inst., Seattle, Wash., 1972)", (H Bass, editor), Lecture Notes in Math. 341, Springer, Berlin (1973) 85-147 MR0338129

[23] C Schlichtkrull, Units of ring spectra and their traces in algebraic $K$-theory, Geom. Topol. 8 (2004) 645-673 MR2057776

[24] C Schommer-Pries, The classification of two-dimensional extended topological field theories, preprint (2011) Available at http://sites.google.com/site/ chrisschommerpriesmath/Home

[25] G Segal, Categories and cohomology theories, Topology 13 (1974) 293-312 MR0353298

[26] N Shimada, K Shimakawa, Delooping symmetric monoidal categories, Hiroshima Math. J. 9 (1979) 627-645 MR549667 
[27] M Shulman, Constructing symmetric monoidal bicategories arXiv:1004.0993v1

[28] R Street, Fibrations in bicategories, Cahiers Topologie Géom. Différentielle 21 (1980) 111-160 MR574662

[29] R Street, Categorical structures, from: "Handbook of algebra, Vol. 1", (M Hazewinkel, editor), North-Holland, Amsterdam (1996) 529-577 MR1421811

[30] R W Thomason, Homotopy colimits in the category of small categories, Math. Proc. Cambridge Philos. Soc. 85 (1979) 91-109 MR510404

[31] R W Thomason, Symmetric monoidal categories model all connective spectra, Theory Appl. Categ. 1 (1995) 78-118 MR1337494

Department of Mathematics, University of Chicago

5734 S University Ave, Chicago IL 60637, USA

aosorno@math. uchicago.edu

http://math.uchicago.edu/ aosorno

Received: 8 December $2010 \quad$ Revised: 21 November 2011 